Research Article

\title{
Design and Analysis of a Rigid-Flexible Parallel Mechanism for a Neck Brace
}

\author{
Jingfang Liu $\mathbb{D}^{1,2}$ Yanxia Cheng $\mathbb{D}^{1},{ }^{1}$ Shuang Zhang $\mathbb{D}^{1},{ }^{1}$ Zhenxin Lu $\mathbb{D}{ }^{1}$ \\ and Guohua Gao $\mathbb{i D}^{1,2}$ \\ ${ }^{1}$ College of Mechanical Engineering and Applied Electronics Technology, Beijing University of Technology, Beijing, China \\ ${ }^{2}$ Key Laboratory of Advanced Manufacturing Technology, Beijing University of Technology, Beijing, China
}

Correspondence should be addressed to Guohua Gao; ggh0912@126.com

Received 3 July 2019; Revised 7 September 2019; Accepted 25 September 2019; Published 3 November 2019

Academic Editor: Xuping Zhang

Copyright ( 92019 Jingfang Liu et al. This is an open access article distributed under the Creative Commons Attribution License, which permits unrestricted use, distribution, and reproduction in any medium, provided the original work is properly cited.

\begin{abstract}
A rigid-flexible parallel mechanism called 3-RXS mechanism as a neck brace for patients with head drooping symptoms (HDS) is presented. The 3-RXS neck brace has a simple and light structure coupled with good rotation performance, so it can be used to assist the neck to achieve flexion and extension, lateral bend, and axial torsion. Firstly, to prove that the X-shaped compliant joint has a rotational degree of freedom (DoF) and can be used in the 3-RRS spherical parallel mechanism (3-RRS SPM), the sixdimensional compliance matrix, axis drift, and DoF of the X-shaped compliant joint have been systematically calculated. Secondly, the 3-RXS mechanism and its pseudo-rigid-body model (PRBM) are obtained by replacing the revolute pair with the $\mathrm{X}$-shaped compliant joint in the 3-RRS SPM. The rotation workspace of the 3-RXS mechanism is also performed. Finally, to verify the rotation function and effect of 3-RXS mechanism for neck-assisted rehabilitation, the kinematics simulations of the 3-RXS and 3-RRS mechanisms are carried out and compared with the theoretical result, and a primary experiment for rotation measurement of 3-RXS mechanism prototype is carried out. All results prove the feasibility of the 3-RXS mechanism for a neck brace.
\end{abstract}

\section{Introduction}

Several neurological diseases such as amyotrophic lateral sclerosis (ALS), Parkinson's disease (PD), and primary lateral sclerosis (PLS) can result in head drooping syndrome (HDS), which is correctable by passive neck extension [1]. The static neck braces are usually used to help patients support their heads. Most of the neck braces consist of an anterior chin support, such as Aspen Vista and Head Master, which causes the patients to have difficulties in breathing and speaking. To improve the breathing capacity, a neck brace using forehead attachment is proposed [2]. Because of the comfort and availability, power wheelchair is a popular brace for patients with HDS, but it is too heavy to carry [3]. A 6-DoF exoskeleton for head and neck motion assist was developed, which can relieve head burden and generate extra head motion assist [4]. A spring-loaded dynamic neck brace can provide restoring forces to the head as it is displaced from the equilibrium configuration [5-7].

However, current braces are mainly static or rigid, which causes difficulty in breathing and has a movement impact resulting in increasing the likelihood of secondary neck injury. Therefore, it is necessary to develop a compliant neck brace. The compliant mechanism is a new type of assemblyfree mechanisms that use compliant joints to replace the joints in conventional mechanisms and utilizes elastic deformations of compliant joints to transmit force or energy [8]. Compliant mechanisms are widely applied in precision instruments, force or displacement amplifiers, flexible micropositioning mechanisms, and medical rehabilitation mechanisms because of its simple structure, no lubrication, no gap, no friction, simple processing, and high precision [9-16]. For example, a modified lever displacement amplifier is proposed for the novel 2-DoFs compliant mechanism design in a piezodriven flexure-based nanopositioning stage [17]. The design and manufacturing processes of a new piezoactuated $X Y$ stage with integrated parallel, decoupled, and stacked kinematics structure for micro/nanopositioning application are presented [18]. In general, the design methods of compliant mechanisms can be divided into rigid body replacement method, degree of freedom and 
constrained spatial topology synthesis (FACT) method, topology optimization method, module method, and so on. The analysis methods of compliant mechanisms can be divided into pseudo-rigid-body model method, structural matrix method, finite element method, and so on [19-22]. Considering simplicity and convenience, the rigid body replacement method is used to obtain a compliant mechanism in this paper. Due to its characteristics of simple structure and good flexibility, the 3-RRS spherical parallel mechanism (SPM) is chosen as the rigid mechanism [23-27].

Many research studies have concerned about the design and analysis of compliant joints. Traditional compliant joints can be commonly divided into two types. One is the notched joint whose integrated design often makes the motion range very small and is more suitable for micromanipulation robot [28-31]. The other is the leaf spring joint mostly used for large deformation [32-34]. Although each of these compliant joints has its own characteristics, it is difficult to meet the requirements of axis drift, stroke, precision, and technological properties at the same time. Most compliant joints have problems such as large axis drift, small offaxis stiffness, complex structure, and so on. $\mathrm{Yu}$ and $\mathrm{Li}$ designed and fabricated an X-shaped compliant joint and applied it to a 3-RRR plane mechanism for experimental research [35]. It was concluded that the X-shaped compliant joint has the advantages of large rotation angle, small axis drift, and simple structure, and it could be used to replace a revolute pair in a plane mechanism.

However, $\mathrm{Yu}$ and Li had not made a specific theoretical analysis for the $\mathrm{X}$-shaped compliant joint. Whether the $\mathrm{X}$-shaped compliant joint can be applied to more compliant mechanisms, especially spatial mechanisms, needs further verification. Therefore, this paper attempts to verify that the $\mathrm{X}$-shaped compliant joint can be applied to a spatial 3-RRS parallel mechanism for a neck brace, and it still has the advantages of large rotation angle and small axis drift. Therefore, the X-shaped compliant joint is used to replace the revolute pair connecting the active and passive rods in a 3-RRS SPM in this paper.

The main line of this paper is as follows: (i) the compliance matrix, axis drift, and degree of freedom of the $\mathrm{X}$-shaped compliant joint are firstly derived and verified, which decide whether $\mathrm{X}$-shaped compliant joint can be used to replace the intermediate revolute pair of the 3-RRS spatial mechanism; (ii) the 3-RXS mechanism for a neck brace is designed and analyzed, where the rotation workspace and kinematics simulation are carried out to validate the feasibility in assisting the neck flexion and extension, lateral bending, and axial torsion; (iii) the effect of the 3-RXS mechanism prototype for neck-assisted rehabilitation is preliminarily shown; and (iv) the conclusion is given.

\section{Analysis of X-Shaped Compliant Joint}

Since the actual function of large rotation angle and small axis drift of the X-shaped compliant joint had been confirmed from experiment in a 3-RRR planar mechanism [35], this paper mainly focuses on its function in a spatial mechanism from theory and simulation.

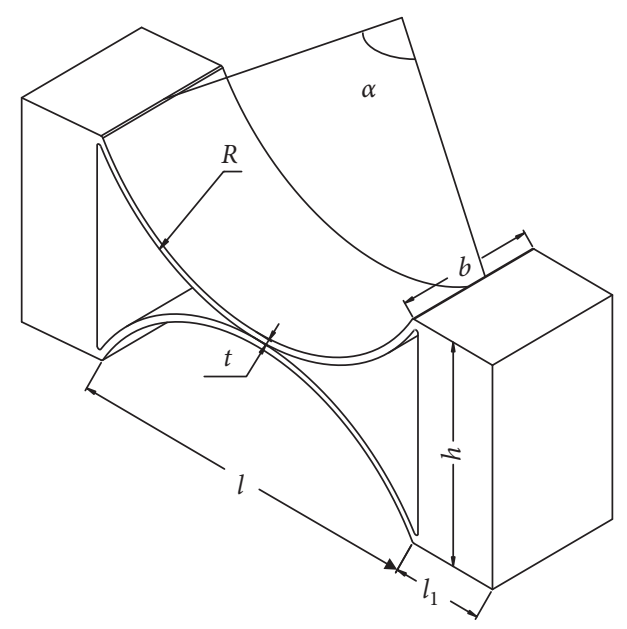

Figure 1: X-shaped compliant joint.

Referring to Figure 1, X-shaped compliant joint is mainly composed of two equal sheets whose centers are fixed together. According to the rule that the position of the rotation center of the revolute pair after the replacement is the same as before, the structural parameters of the $\mathrm{X}$-shaped compliant joint are chosen as follows: $R=10.5 \mathrm{~mm}, \alpha=120^{\circ}, b=6 \mathrm{~mm}$, $t=1 \mathrm{~mm}, l=16.45 \mathrm{~mm}, l_{1}=4.13 \mathrm{~mm}$, and $h=11.5 \mathrm{~mm}$.

$\mathrm{Yu}$ and Li mainly carried out structural design and experimental measurement of the axis drift on the X-shaped compliant joint. They found that when the X-shaped compliant joint rotates from $-45^{\circ}$ to $45^{\circ}$, the position error of the rotation center is only $0.3717 \mathrm{~mm}$, and the precision is relatively high.

The six-dimensional compliance matrix, axis drift, and DoF of the X-shaped compliant joint need to be calculated systematically to verify whether the X-shaped compliant joint has a rotational DoF and small axis drift, which decide whether it can be applied to replace the R-joint in the 3-RRS spatial mechanism.

At present, the research on the spatial compliance matrix of the leaf spring joint is still few. In order to apply the $\mathrm{X}$-shaped compliant joint to the spatial mechanism, this paper uses the integral method to derive the six-dimensional compliance matrix for the $\mathrm{X}$-shaped leaf spring compliant joint. This paper solves out the compliance matrix of a quarter curved plate firstly and then connects all quarter curved plates in series and parallel to get the whole sixdimensional compliance matrix of the X-shaped compliant joint. In addition, the DoF of the X-shaped compliant joint is analyzed based on the compliance matrix.

2.1. Calculation and Analysis of the Compliance Matrix. The beam deformation theory is used to calculate the compliance matrix of the X-shaped compliant joint. As shown in Figure 2, the X-shaped compliant joint can be regarded as a cantilever beam with one end fixed and the other end free, which can be bent, axially stretched, sheared, and turned round subjected to the action of the six-dimensional unit force $F$.

The relationship between force and deformation can be expressed as 


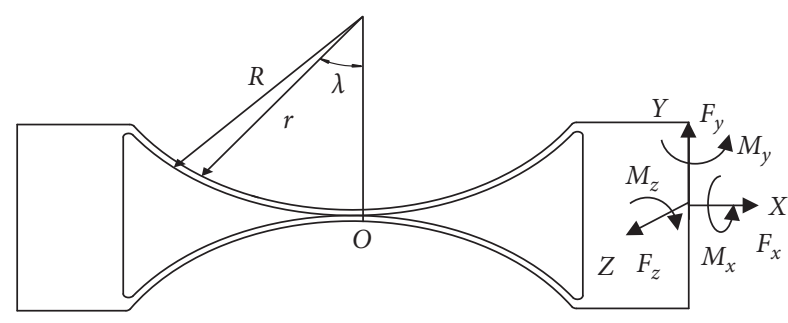

FIGURE 2: X-shaped compliant joint.

$$
X=C_{X} F
$$

where $F$ is the six-dimensional unit force, $X$ is the six-dimensional deformation, and $C_{X}$ is the compliance matrix of the X-shaped compliant joint:

$$
\begin{aligned}
& F=\left[F_{x}, F_{y}, F_{z}, M_{x}, M_{y}, M_{z}\right]^{T}, \\
& X=\left[\Delta x, \Delta y, \Delta z, \alpha_{x}, \alpha_{y}, \alpha_{z}\right]^{T} .
\end{aligned}
$$

As shown in Figure 2, the coordinate system $X Y Z$ is established at the end of the X-shaped compliant joint, and the compliance matrix can be expressed as follows:

$$
C_{X}=\left[\begin{array}{cccccc}
\frac{\Delta_{x}}{F_{x}} & 0 & 0 & 0 & 0 & 0 \\
0 & \frac{\Delta_{y}}{F_{y}} & 0 & 0 & 0 & \frac{\Delta_{y}}{M_{z}} \\
0 & 0 & \frac{\Delta_{z}}{F_{z}} & 0 & \frac{\Delta_{z}}{M_{y}} & 0 \\
0 & 0 & 0 & \frac{\alpha_{x}}{M_{x}} & 0 & 0 \\
0 & 0 & \frac{\alpha_{y}}{F_{z}} & 0 & \frac{\alpha_{y}}{M_{y}} & 0 \\
0 & \frac{\alpha_{z}}{F_{y}} & 0 & 0 & 0 & \frac{\alpha_{z}}{M_{z}}
\end{array}\right] .
$$

The X-shaped compliant joint can be regarded as composed of the left half and the right half in series. The left half and the right half are composed of two quarters of the curved plates in parallel.

Firstly, the compliance matrix of the quarter of the curved plate is derived. As shown in Figure 3, a coordinate system $o_{1^{-}}$ $x_{1} y_{1} z_{1}$ is established at the reference point $o_{1}$ of the quarter of the curved plate, and the compliance matrix can be expressed as

$$
C_{1 / 4}=\left[\begin{array}{cccccc}
C_{11} & 0 & 0 & 0 & 0 & 0 \\
0 & C_{22} & 0 & 0 & 0 & C_{62} \\
0 & 0 & C_{33} & 0 & C_{53} & 0 \\
0 & 0 & 0 & C_{44} & 0 & 0 \\
0 & 0 & C_{35} & 0 & C_{55} & 0 \\
0 & C_{26} & 0 & 0 & 0 & C_{66}
\end{array}\right]^{T}
$$

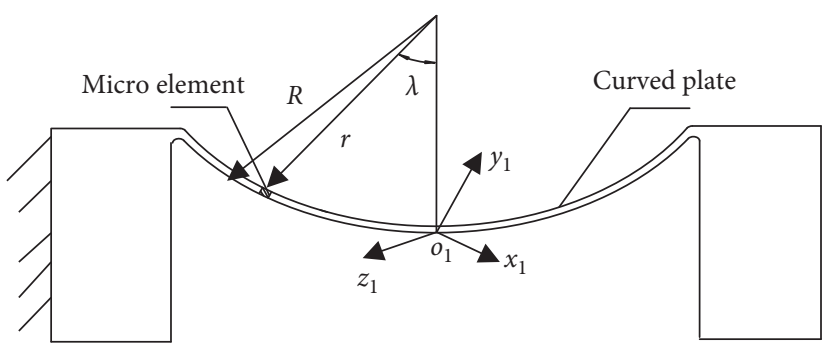

(a)

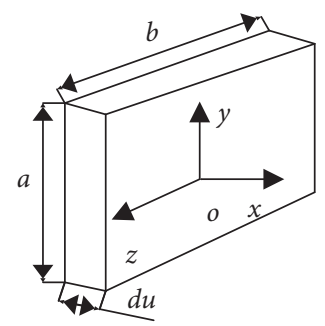

(b)

Figure 3: (a) Half of the X-shaped compliant joint. (b) Cross section.

Each item in equation (4) is derived from the beam deformation theory considering the actions of force and moment. The elastic deformation of the compliant joint is mainly realized by the curved plate. It is assumed that the left end of the compliant joint is fixed, and the moment subjected to the rotating center of the compliant joint is $M_{z}$. The angle of the right end around the $z$-axis is $\alpha_{z}$. The deformation is mainly concentrated in the curved, so the deformation of the rest structure is ignored.

A microelement with the rectangular section is taken at the curved. The specific structural dimensions are shown in Figure 3, including height $a$, width $b$, and the thickness $d u$. Since the size of the curved plate is much smaller than the overall structural size of the mechanism, for the convenience of analysis, the moment on each microelement is regarded as a constant $M_{z}$ and the angle of each microelement is $d \alpha_{z}$. The deformation and compliance calculation processes are as follows:

$$
\begin{aligned}
a & =\sqrt{R^{2}-(r \sin \lambda)^{2}}-r \cos \lambda, \\
d u & =d(r \sin \lambda)=r \cos \lambda d \lambda .
\end{aligned}
$$

The angular deformation $d \alpha_{z}$ of the microelement along the $z$-axis under the action of the moment $M_{z}$ is

$$
d \alpha_{z}=\frac{M_{z}}{E I_{z}} d u=\frac{12 M_{z}}{E b\left(\sqrt{R^{2}-(r \sin \lambda)^{2}}-r \cos \lambda\right)^{3}} d u
$$

where $E$ is the modulus of elasticity of the material and $I_{z}$ is the moment of inertia of the microelement along the $z$-axis, which is given as

$$
I_{z}=\frac{b a^{3}}{12}
$$

Therefore, 


$$
\begin{aligned}
C_{66} & =\frac{\alpha_{z}}{M_{z}}=\frac{12 r}{E b} \int_{-\lambda_{m}}^{0} \frac{\cos \lambda}{\left(\sqrt{R^{2}-(r \sin \lambda)^{2}}-r \cos \lambda\right)^{3}} d \lambda \\
& =\frac{12 r}{E b\left(R^{2}-r^{2}\right)^{3}} N_{66} .
\end{aligned}
$$

The angular displacement $\alpha_{z}$ generated by force $F_{y}$ is equivalent to the angular displacement generated by the bending moment $F_{y} r \sin \lambda$, which can be expressed as

$$
d \alpha_{z}=\frac{-F_{y} r \sin \lambda}{E I_{z}} d u=\frac{-12 F_{y} r \sin \lambda}{E b\left(\sqrt{R^{2}-(r \sin \lambda)^{2}}-r \cos \lambda\right)^{3}} d u \text {. }
$$

Therefore,

$$
\begin{aligned}
C_{26} & =\frac{\alpha_{z}}{F_{y}}=\frac{-12 r^{2}}{E b} \int_{-\lambda_{m}}^{0} \frac{\cos \lambda \sin \lambda}{\left(\sqrt{R^{2}-(r \sin \lambda)^{2}}-r \cos \lambda\right)^{3}} d \lambda \\
& =\frac{-12 r^{2}}{E b\left(R^{2}-r^{2}\right)^{3}} N_{26} .
\end{aligned}
$$

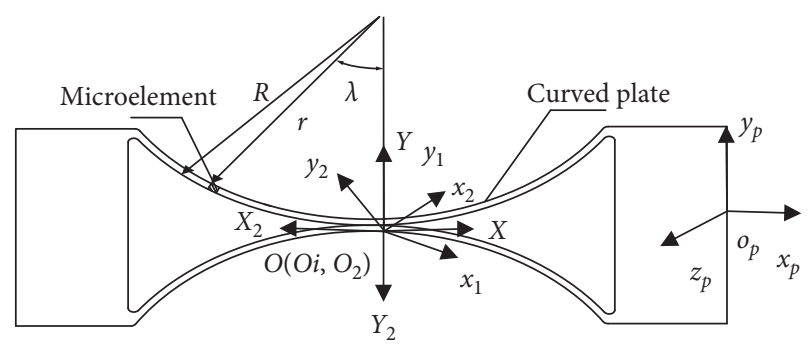

Figure 4: X-shaped compliant joint.

Similarly, other elements in the compliance matrix can also be derived and are shown in the Appendix. The compliance matrix $C_{1 / 4}$ at the end coordinate $o_{1}-x_{1} y_{1} z_{1}$ of the quarter of the curved plate is

\footnotetext{
$\left[\frac{r N_{55}}{E b\left(R^{2}-r^{2}\right)}\right.$

0

$0 \quad \frac{12 r^{3} N_{22}}{E b\left(R^{2}-r^{2}\right)^{3}}+\frac{k r N_{55}}{G b\left(R^{2}-r^{2}\right)}$

0

0

0

0

0

$\frac{12 r^{2} N_{35}}{E b^{3}\left(R^{2}-r^{2}\right)}$

$0 \quad \frac{-12 r^{2} N_{26}}{E b\left(R^{2}-r^{2}\right)^{3}}$

0

where $N_{66}, N_{26}, N_{55}, N_{35}, N_{22}$, and $N_{33}$ are shown in the

If the joint is divided into two identical parts from left to right, each part can be regarded as the connection of upperleft semicurved plate and lower-left semicurved plate in parallel. As shown in Figure 4, four coordinate systems are set up, where $o_{1}-x_{1} y_{1} z_{1}$ and $o_{2}-x_{2} y_{2} z_{2}$ are the coordinate systems of the upper-left semicurved plate and lower-left semicurved plate, and $O-X Y Z$ and $\mathrm{O}_{2}-X_{2} Y_{2} Z_{2}$ are those of the left part and the right part, respectively.
}

$\frac{12 r^{3} N_{33}}{E b^{3}\left(R^{2}-r^{2}\right)}+\frac{k r N_{55}}{G b\left(R^{2}-r^{2}\right)}$ Appendix.

$$
\left.\begin{array}{ccc}
0 & 0 & 0 \\
0 & 0 & \frac{-12 r^{2} N_{26}}{E b\left(R^{2}-r^{2}\right)^{3}} \\
\frac{12 N_{55} N_{35}}{E b\left(R^{2}-r^{2}+b^{2}\right)\left(R^{2}-r^{2}\right)} & 0 \\
0 & \frac{12 r N_{55}}{E b^{3}\left(R^{2}-r^{2}\right)} & 0 \\
0 & 0 & \frac{12 r N_{66}}{E b\left(R^{2}-r^{2}\right)^{3}}
\end{array}\right]^{T}
$$

The compliance matrix of the left part of the X-shaped compliant joint in the central reference coordinate system $O-X Y Z$ is

$$
C_{1 / 2}=\left[\left(A d_{1} C_{1 / 4} A d_{1}^{T}\right)^{-1}+\left(A d_{2} C_{1 / 4} A d_{2}^{T}\right)^{-1}\right]^{-1},
$$

where $A d_{1}$ is the transformation matrix from the coordinate system $o_{1}-x_{1} y_{1} z_{1}$ to $O-X Y Z$ and $A d_{2}$ is the transformation matrix from the coordinate system $o_{2}-x_{2} y_{2} z_{2}$ to $O-X Y Z$, which are given as follows: 


$$
\begin{aligned}
& A d_{1}=\left[\begin{array}{cc}
R_{1} & t_{1} R_{1} \\
0 & R_{1}
\end{array}\right] \text {, } \\
& A d_{2}=\left[\begin{array}{cc}
R_{2} & t_{2} R_{2} \\
0 & R_{2}
\end{array}\right] \text {, } \\
& R_{1}=\left[\begin{array}{ccc}
\cos \theta_{1} & -\sin \theta_{1} & 0 \\
\sin \theta_{1} & \cos \theta_{1} & 0 \\
0 & 0 & 1
\end{array}\right] \text {, } \\
& R_{2}=\left[\begin{array}{ccc}
\cos \theta_{2} & -\sin \theta_{2} & 0 \\
\sin \theta_{2} & \cos \theta_{2} & 0 \\
0 & 0 & 1
\end{array}\right] \text {, } \\
& t_{1}=\left[\begin{array}{ccc}
0 & 0 & \frac{t}{2} \\
0 & 0 & 0 \\
t & 0 & 0
\end{array}\right] \text {, } \\
& t_{2}=\left[\begin{array}{ccc}
0 & 0 & -\frac{t}{2} \\
0 & 0 & 0 \\
\frac{t}{2} & 0 & 0
\end{array}\right] \text {, } \\
& \theta_{1}=\frac{\lambda_{m}}{2}=30^{\circ} \text {, } \\
& \theta_{2}=-\frac{\lambda_{m}}{2}=-30^{\circ} \text {. }
\end{aligned}
$$

The whole $\mathrm{X}$-shaped compliant joint is the connection of two parts in series, and the compliance matrix of the $\mathrm{X}$-shaped compliant joint in the end reference coordinate system $o_{p}-x_{p} y_{p} z_{p}$ is

$$
C_{X}=A d_{3} C_{1 / 2} A d_{3}^{T}+A d_{4} C_{1 / 2} A d_{4}^{T},
$$

where $A d_{3}$ is the transformation matrix from the coordinate system $O-X Y Z$ to $o_{p}-x_{p} y_{p} z_{p}$ and $A d_{4}$ is the transformation matrix from the coordinate system $\mathrm{O}_{2}-X_{2} Y_{2} Z_{2}$ to $o_{p}-x_{p} y_{p} z_{p}$, which are given by

$$
\begin{aligned}
A d_{3} & =\left[\begin{array}{ll}
I_{3} & t_{3} \\
0 & I_{3}
\end{array}\right], \\
A d_{4} & =\left[\begin{array}{ll}
R_{4} & R_{4} t_{4} \\
0 & R_{4}
\end{array}\right], \\
t_{3} & =t_{4}=\left[\begin{array}{ccc}
0 & 0 & 0 \\
0 & 0 & r \sin \lambda_{m}+l_{1} \\
0 & -\left(r \sin \lambda_{m}+l_{1}\right) & 0
\end{array}\right], \\
R_{4} & =\left[\begin{array}{ccc}
\cos \theta_{4} & -\sin \theta_{4} & 0 \\
\sin \theta_{4} & \cos \theta_{4} & 0 \\
0 & 0 & 1
\end{array}\right], \\
\theta_{4} & =\pi .
\end{aligned}
$$

Therefore, the rotational compliance of the X-shaped compliant joint around the $z$-axis is

$$
C_{z}=\frac{\alpha_{z}}{M_{z}}=\frac{C_{11} C_{66} \cos ^{2}\left(\lambda_{m} / 2\right)+C_{22} C_{66} \sin ^{2}\left(\lambda_{m} / 2\right)-C_{26}^{2} \sin ^{2}\left(\lambda_{m} / 2\right)}{C_{11} \cos ^{2}\left(\lambda_{m} / 2\right)+C_{22} \sin ^{2}\left(\lambda_{m} / 2\right)+\left(C_{66} t^{2} / 4\right)-t C_{26} \sin \left(\lambda_{m} / 2\right)}
$$

It can be seen from equation (17) that the rotational compliance $C_{z}$ of the X-shaped compliant joint around the $z$ axis is related to the width $(b)$, the inner diameter $(r)$, the outer diameter $(R)$, the thickness $(t)$, and the half of the center angle and the material. The relationship between the structural parameters of the curved plate and the rotational compliance $C_{z}$ of the X-shaped compliant joint is analyzed and is shown in Figure 5.

It can be seen that the rotational compliance of the $\mathrm{X}$-shaped compliant joint decreases as the width $b$ and the thickness $t$ of the curved plate increase. The rotational compliance increases with increasing the half of the central angle $\lambda_{m}$. The thickness $t$ and the half of the central angle $\lambda_{m}$ have a greater influence on the rotational compliance than the width $b$. It is because $t$ and $\lambda_{m}$ determine the size of the weakest structure of the compliant joint, where it has the greatest influence on the rotational compliance of the $\mathrm{X}$-shaped compliant joint.

The relationship between the rotational compliance and the structural parameters of the X-shaped compliant joint is obtained through the analysis of the compliance matrix, which is helpful for the optimization of joint structural parameters.

2.2. Calculation and Analysis of the Axis Drift. When the end of the X-shaped compliant joint is only acted on $M_{z}$, the radius of the parasitic error circle is used to analyze the axis drift, and equation (15) can be further simplified to 


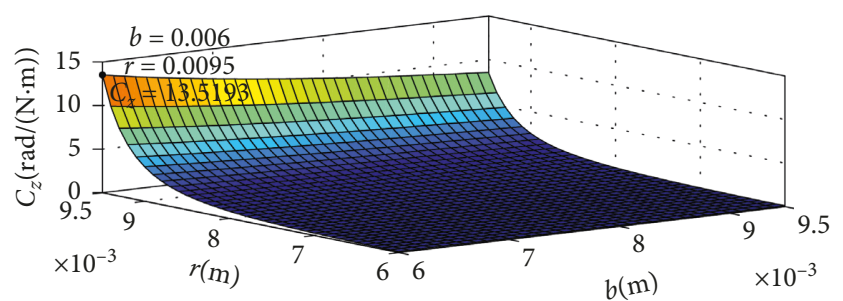

(a)

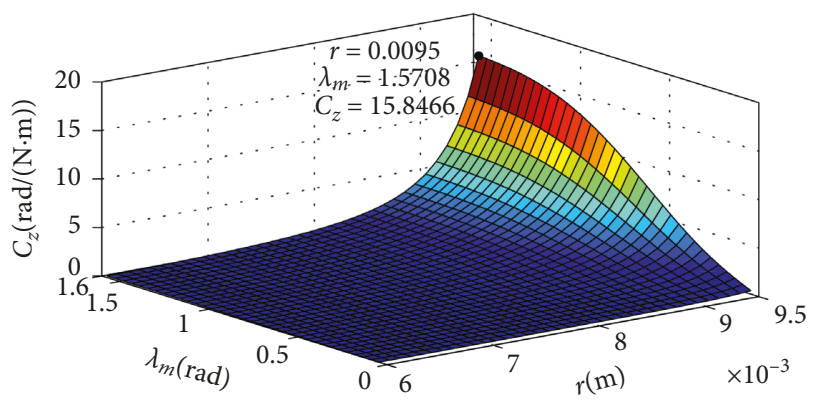

(c)

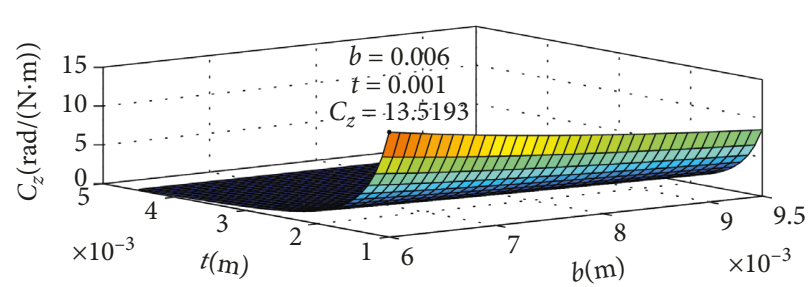

(b)

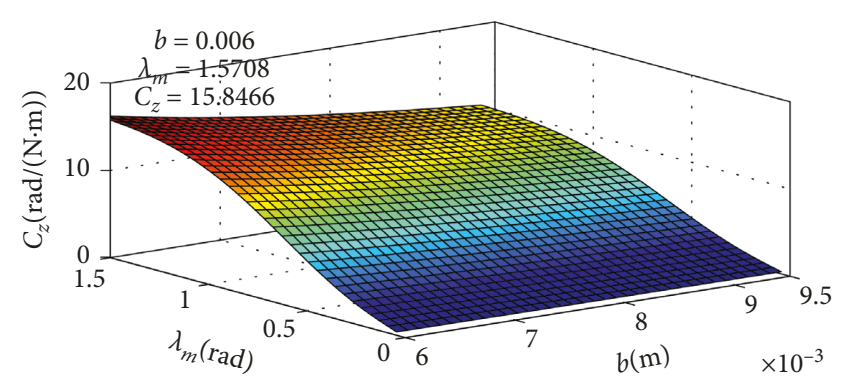

(d)

Figure 5: The variation of rotational compliance $C_{z}$ with the structural parameters $b, r, t$, and $\lambda_{m}$. (a) The variation of rotational compliance with $b$ and $r$. (b) The variation of rotational compliance with $b$ and $t$. (c) The variation of rotational compliance with $\lambda_{m}$ and $r$. (d) The variation of rotational compliance with $b$ and $\lambda_{m}$.

$$
\begin{aligned}
& \theta_{z}=\frac{\lambda_{m}(R+r)}{E I_{z}}\left(C_{66}^{X} M_{z}\right), \\
& \delta_{y}=\frac{\lambda_{m}(R+r)}{E I_{z}}\left(C_{62}^{X} M_{z}\right) .
\end{aligned}
$$

According to equations (18) and (19), it can be seen that the X-shaped compliant joint will generate parasitic motion $\delta_{y}$ while generating the rotation $\theta_{z}$ in functional direction under the action of $M_{z}$. Furthermore, the radius of the parasitic motion error circle in the $x$-axis direction is obtained as follows:

$$
R_{x-M_{z}}=\frac{\delta y}{\theta_{z}}=\frac{C_{62}^{X}}{C_{66}^{X}}
$$

where

$$
\begin{aligned}
C_{66}^{X} & =\frac{C_{11} C_{66} \cos ^{2}\left(\lambda_{m} / 2\right)+C_{22} C_{66} \sin ^{2}\left(\lambda_{m} / 2\right)-C_{26}^{2} \sin ^{2}\left(\lambda_{m} / 2\right)}{C_{11} \cos ^{2}\left(\lambda_{m} / 2\right)+C_{22} \sin ^{2}\left(\lambda_{m} / 2\right)+\left(C_{66} t^{2} / 4\right)-t C_{26} \sin \left(\lambda_{m} / 2\right)}, \\
C_{62}^{X} & =\frac{\left(r \sin \lambda_{m}+413 / 10^{5}\right)\left(C_{11} C_{66} \cos ^{2}\left(\lambda_{m} / 2\right)+C_{22} C_{66} \sin ^{2}\left(\lambda_{m} / 2\right)-C_{26} C_{62} \sin \sin ^{2}\left(\lambda_{m} / 2\right)\right)}{\left(C_{11} \cos ^{2}\left(\lambda_{m} / 2\right)+C_{22} \sin ^{2}\left(\lambda_{m} / 2\right)+\left(C_{66} t^{2} / 4\right)-C_{26} t \sin \left(\lambda_{m} / 2\right)\right)} .
\end{aligned}
$$

The ideal center of the parasitic error circle is the intersection of the two curved plates, and the radius is $r \sin \lambda_{m}+l_{1}$. For the convenience of analysis, the radius of the parasitic error circle in the $x$-axis, expressed as equation (20), is dimensionalized to one, that is,

$$
\begin{aligned}
r_{x-M_{z}}= & \left|R_{x-M_{z}} \lambda_{m}\right|=\frac{\delta y \lambda_{m}}{\theta_{z}}=\frac{\lambda_{m} C_{62}^{X}}{C_{66}^{X}} \\
= & \frac{\lambda_{m}\left(r \sin \lambda_{m}+413 / 10^{5}\right)\left(C_{11} C_{66} \cos ^{2}\left(\lambda_{m} / 2\right)+C_{22} C_{66} \sin ^{2}\left(\lambda_{m} / 2\right)-C_{26} C_{62} \sin ^{2}\left(\lambda_{m} / 2\right)\right)}{\left(C_{11} \cos ^{2}\left(\lambda_{m} / 2\right)+C_{22} \sin ^{2}\left(\lambda_{m} / 2\right)+\left(C_{66} t^{2} / 4\right)-C_{26} t \sin \left(\lambda_{m} / 2\right)\right)} \\
& * \frac{\left(C_{11} \cos ^{2}\left(\lambda_{m} / 2\right)+C_{22} \sin ^{2}\left(\lambda_{m} / 2\right)+\left(C_{66} t^{2} / 4\right)-t C_{26} \sin \left(\lambda_{m} / 2\right)\right)}{\left(C_{11} C_{66} \cos ^{2}\left(\lambda_{m} / 2\right)+C_{22} C_{66} \sin ^{2}\left(\lambda_{m} / 2\right)-C_{26}^{2} \sin ^{2}\left(\lambda_{m} / 2\right)\right)} .
\end{aligned}
$$


Therefore, the total radius of the parasitic error circle in the $x$-axis direction is

$$
\begin{aligned}
r_{x-M_{z}}^{1}= & r \sin \lambda_{m}+l_{1}-p_{r z} \\
= & r \sin \lambda_{m}+l_{1}-\left[\frac{\lambda_{m}\left(r \sin \lambda_{m}+413 / 10^{5}\right)\left(C_{11} C_{66} \cos ^{2}\left(\lambda_{m} / 2\right)+C_{22} C_{66} \sin ^{2}\left(\lambda_{m} / 2\right)-C_{26} C_{62} \sin \sin ^{2}\left(\lambda_{m} / 2\right)\right)}{\left(C_{11} \cos ^{2}\left(\lambda_{m} / 2\right)+C_{22} \sin ^{2}\left(\lambda_{m} / 2\right)+\left(C_{66} t^{2} / 4\right)-C_{26} t \sin \left(\lambda_{m} / 2\right)\right)}\right. \\
& \left.* \frac{\left(C_{11} \cos ^{2}\left(\lambda_{m} / 2\right)+C_{22} \sin ^{2}\left(\lambda_{m} / 2\right)+\left(C_{66} t^{2} / 4\right)-t C_{26} \sin \left(\lambda_{m} / 2\right)\right)}{\left(C_{11} C_{66} \cos ^{2}\left(\lambda_{m} / 2\right)+C_{22} C_{66} \sin ^{2}\left(\lambda_{m} / 2\right)-C_{26}^{2} \sin ^{2}\left(\lambda_{m} / 2\right)\right)}\right] .
\end{aligned}
$$

When the width $b$ of the curved plate is $6 \mathrm{~mm}$, take the thickness $t$ of the curved plate as $0.8 \mathrm{~mm}, 1.0 \mathrm{~mm}, 1.2 \mathrm{~mm}$, and $1.5 \mathrm{~mm}$, respectively and obtain the relationship curve, which represents the variation of the radius of the parasitic error circle with the half of the center angle $\lambda_{m}$ and thickness $t$, as shown in Figure 6.

It is obvious that

(1) The parasitic error circle radius of the X-shaped compliant joint is independent of the thickness $t$ of the curved plate.

(2) The parasitic error circle radius of the X-shaped compliant joint first increases and then decreases with the increase of the half-central angle $\lambda_{m}$ of the curved plate. When $\lambda_{m}$ is $0.9874 \mathrm{rad}$, that is, about $56.5716^{\circ}$, the radius of the parasitic error circle decreases to the minimum value $0.14717 \mathrm{~mm}$, and the $\mathrm{X}$-shaped compliant joint has the highest rotation precision.

In summary, the thicker the $\mathrm{X}$-shaped compliant joint is, the larger the central angle it has, and the greater the rotational compliance is. The axis drift is the smallest, and the $\mathrm{X}$-shaped compliant joint has the highest rotation precision when half of the central angle is about $56.5716^{\circ}$.

Therefore, take half of the central angle $\lambda_{m}$ and the thickness $t$ of the curved plate as $60^{\circ}$ and $1 \mathrm{~mm}$, respectively. The structural parameters of the X-shaped compliant joint are shown in Table 1.

Calculate equations (1) (15), and the compliance matrix of the X-shaped compliant joint can be obtained as follows:

$$
C_{X}=\left[\begin{array}{cccccc}
3.0424 \times 10^{-6} & 0 & 0 & 0 & 0 & 0 \\
0 & 2.0864 \times 10^{-3} & 0 & 0 & 0 & 1.6706 \times 10^{-1} \\
0 & 0 & 1.5236 \times 10^{-4} & 0 & -1.0639 \times 10^{-2} & 0 \\
0 & 0 & 0 & 9.6228 \times 10^{-1} & 0 & 0 \\
0 & 0 & -1.0639 \times 10^{-2} & 0 & 8.6096 \times 10^{-1} & 0 \\
0 & 1.6706 \times 10^{-1} & 0 & 0 & 0 & 1.3519 \times 10^{1}
\end{array}\right] .
$$

2.3. Calculation and Analysis of the Degree of Freedom. The compliance matrix of the X-shaped compliant joint lays a foundation for the following calculation and analysis of the DoF of the X-shaped compliant joint. Among the six main diagonal elements in equation (24), the dimension of the mobile compliances $\Delta_{x} / F_{x}, \Delta_{y} / F_{y}$, and $\Delta_{z} / F_{z}$ is one, and the dimension of the rotational compliances $\alpha_{x} / M_{x}, \alpha_{y} / M_{y}$, and $\alpha_{z} /$
$M_{z}$ is $L^{2}$, which cannot be directly compared. Based on the compliance matrix, the degree of freedom of the X-shaped compliant joint is calculated by the analytical method proposed by Yu et al. [36].

The eigenvalues and eigenvectors of the compliance matrix $C_{X}$ expressed as equation (24) are calculated as follows:

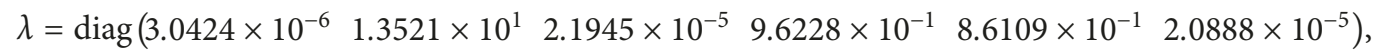

$$
\begin{aligned}
& V=\left[\begin{array}{cccccc}
1 & 0 & 0 & 0 & 0 & 0 \\
0 & -1.2356 \times 10^{-2} & 1 & 0 & 0 & 0 \\
0 & 0 & 0 & 0 & 1.2357 \times 10^{-2} & -1 \\
0 & 0 & 0 & 1 & 0 & 0 \\
0 & 0 & 0 & 0 & -1 & -1.2357 \times 10^{-2} \\
0 & -1 & -1.2356 \times 10^{-2} & 0 & 0 & 0
\end{array}\right] .
\end{aligned}
$$




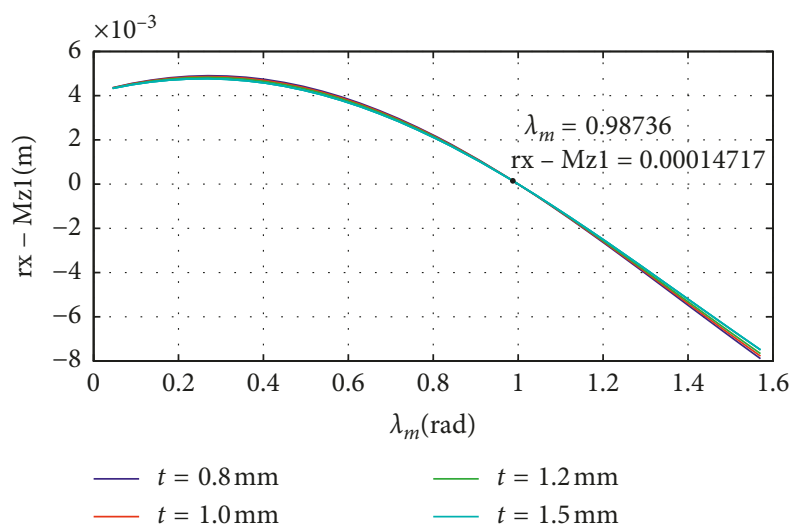

FIgURE 6: The radius of parasitic error circle varying with $\lambda_{m}$ and $t$.

TABLE 1: Structural parameters of the X-shaped compliant joint.

\begin{tabular}{lccccc}
\hline Outer diameter & Central angle & Section & Boss length & Boss height & Elastic modulus \\
\hline$R(\mathrm{~mm})$ & $2 \lambda_{m}\left({ }^{\circ}\right)$ & $(b \times t)\left(\mathrm{mm}^{2}\right)$ & $l_{1}(\mathrm{~mm})$ & $h(\mathrm{~mm})$ & $E(\mathrm{MPa})$ \\
10.5 & 120 & $6 \times 1$ & 4.13 & 11.5 & 520 \\
\hline
\end{tabular}

The rotational compliance is divided by $l /\left(E I_{z}\right)$, while the mobile compliance is divided by $l^{3} /\left(E I_{z}\right)$

$$
\lambda=\operatorname{diag}\left(8.6102 \times 10^{-5} \quad 1.6785 \times 10^{-1} \quad 6.2106 \times 10^{-4} \quad 1.1946 \times 10^{-2} \quad 1.0690 \times 10^{-2} 5.9115 \times 10^{-4}\right) .
$$

The ratio of each eigenvalue $\lambda_{i}$ to $\lambda_{\max }$ can be calculated. If the value of $\left|\lambda_{i} / \lambda_{\max }\right|$ is far less than 1 , the eigenvalue $\lambda_{i}$ is assigned a value of zero:

$$
\begin{aligned}
\lambda_{\max }= & 1.6785 \times 10^{-1}, \\
\lambda_{1}= & 1.1946 \times 10^{-2}, \\
\lambda_{2}= & 1.0690 \times 10^{-2}, \\
\lambda_{3}= & 6.2106 \times 10^{-4}, \\
\lambda_{4}= & 5.9115 \times 10^{-4}, \\
\lambda_{5}= & 8.6102 \times 10^{-5}, \\
\frac{\lambda_{1}}{\lambda_{\max }}= & \frac{1.1946 \times 10^{-2}}{1.6785 \times 10^{-1}}, \\
\frac{\lambda_{2}}{\lambda_{\max }}= & \frac{1.0690 \times 10^{-2}}{1.6785 \times 10^{-1}}, \\
\frac{\lambda_{3}}{\lambda_{\max }}= & \frac{6.2106 \times 10^{-4}}{1.6785 \times 10^{-1}}, \\
\frac{\lambda_{4}}{\lambda_{\max }}= & \frac{5.9115 \times 10^{-4}}{1.6785 \times 10^{-1}}, \\
\frac{\lambda_{5}}{\lambda_{\max }}= & \frac{8.6102 \times 10^{-5}}{1.6785 \times 10^{-1}}, \\
\lambda= & \operatorname{diag}\left(3.0424 \times 10^{-6} \quad 1.3521 \times 10^{1} \quad 2.1945 \times 10^{-5} \quad 9.6228 \times 10^{-1} \quad 8.6109 \times 10^{-1} \quad 2.0888 \times 10^{-5}\right) \\
\approx & \operatorname{diag}\left(0 \quad 1.3521 \times 10^{1} \quad 0 \quad 0 \quad 0\right) .
\end{aligned}
$$


After processing, the number of nonzero eigenvalue is the number of degree of freedom of the X-shaped compliant joint. So, the X-shaped compliant joint has one degree of freedom.

The eigenvectors corresponding to zero eigenvalue are found out and form the constrained screw space of the $\mathrm{X}$-shaped compliant joint:

$$
W=\left[\begin{array}{ccccc}
1 & 0 & 0 & 0 & 0 \\
0 & 1 & 0 & 0 & 0 \\
0 & 0 & 0 & 1.2357 \times 10^{-2} & -1 \\
0 & 0 & 1 & 0 & 0 \\
0 & 0 & 0 & -1 & -1.2357 \times 10^{-2} \\
0 & -1.2356 \times 10^{-2} & 0 & 0 & 0
\end{array}\right] .
$$

According to duality of freedom space and constrained space, the freedom space of the X-shaped compliant joint can be obtained as

$$
T=\left(0,0,1 ; 0,1.2356 \times 10^{-2}, 0\right),
$$

which shows that the $\mathrm{X}$-shaped compliant joint has a rotational degree of freedom, whose axis is parallel to the $z$ axis, and passes through the point $\left(-1.2356 \times 10^{-2}, 0,0\right)^{T}$. Note that the coordinate of point $O$ is $\left(-r \times \sin \lambda_{m}-l_{1}, 0,0\right)^{T}$, which equals $\left(-1.2357 \times 10^{-2}, 0,0\right)^{T}$.
The coordinate of the rotation center calculated theoretically is basically consistent with that of the ideal model. The relative error is shown as follows:

$$
\delta=\frac{|12.356-12.3572|}{12.3572} \times 100 \%=0.0097 \% .
$$

This error is mainly due to the integral calculation. Therefore, the rotation axis of the $\mathrm{X}$-shaped compliant joint is considered to be along the $z$-axis and passes through point O.

\section{ANSYS Simulation of X-Shaped Compliant Joint}

ANSYS Workbench is used to develop a model for the $\mathrm{X}$-shaped compliant joint. The elastic modulus is $520 \mathrm{MPa}$, and Poisson's ratio is 0.4. A hexahedral mesh is created for the X-shaped compliant joint model, and a fixed constraint is added to the left end. The unit force of $F_{x}=1 \mathrm{~N}, F_{y}=1 \mathrm{~N}$, and $F_{z}=1 \mathrm{~N}$ and the unit torque of $M_{x}=1 \mathrm{~N} \cdot \mathrm{m}, M_{y}=1 \mathrm{~N} \cdot \mathrm{m}$, and $M_{z}=1 \mathrm{~N} \cdot \mathrm{m}$ are added, respectively, at the center of the right end. After loading, the X-shaped compliant joint is fixed at the left end and rotated at the right end. The compliance matrix of the X-shaped compliant joint is obtained as

$$
C_{X_{e}}=\left[\begin{array}{cccccc}
3.0837 \times 10^{-6} & 0 & 0 & 0 & 0 & 0 \\
0 & 2.1845 \times 10^{-3} & 0 & 0 & 0 & 1.6921 \times 10^{-1} \\
0 & 0 & 1.5946 \times 10^{-4} & 0 & -1.1915 \times 10^{-2} & 0 \\
0 & 0 & 0 & 1.0690 & 0 & 0 \\
0 & 0 & -1.1915 \times 10^{-2} & 0 & 9.0398 \times 10^{-1} & 0 \\
0 & 1.6921 \times 10^{-1} & 0 & 0 & 0 & 1.4037 \times 10^{1}
\end{array}\right] .
$$

The comparison of theoretical calculations with finite element simulation results is shown in Table 2.

It is found that the theoretical calculation results of the compliance matrix are basically consistent with the ANSYS simulation results under the same structural parameters, and the maximum error is $10.709 \%$, which demonstrates the correctness of the compliance formula. The theoretical results are slightly different, which is attributed to the simplified model in theoretical calculation, where only the deformation of the curved part is considered. So, the rotational compliance obtained by theory and simulation will be slightly biased.

The maximum rotation angle connecting the active rod and the passive rod is about $28^{\circ}$. So as the replacement joint, the maximum rotation angle of the X-shaped compliant joint is designed as $30^{\circ}$. ANSYS simulation is used to verify whether the X-shaped compliant joint would rotate $30^{\circ}$. When the $\mathrm{X}$-shaped compliant joint rotates $30^{\circ}$, the required torque is as follows:

$$
M_{z}=\frac{\alpha}{C_{z_{e}}}=\frac{\pi}{6 \times 14.037}=37.3013 \mathrm{~N} \cdot \mathrm{mm} .
$$

In the ANSYS Workbench, the X-shaped compliant joint is fixed at the left end, and the right end is applied to the bending moment $M_{z}$ around the $z$-axis for simulation. The result is shown in Figure 7.

From Figure 7, it is clear that when the $\mathrm{X}$-shaped compliant joint rotates $30^{\circ}$, the maximum stress is $23.477 \mathrm{MPa}$, which occurs at the connection of two curved plates, and the safety factor is 1.461 . In addition, through the finite element simulation, the $\mathrm{X}$-shaped compliant joint can rotate about $43^{\circ}$ at the maximum.

To verify the DoF of the X-shaped compliant joint, the first six-order natural frequency and mode shapes of the $\mathrm{X}$-shaped compliant joint are obtained by the modal simulation, as shown in Figure 8 and Table 3, which determine the number and the nature of DoF of the X-shaped compliant joint, respectively. 
TABLE 2: The relation between external load and deformation.

\begin{tabular}{lcccccccc}
\hline Deformation & $\begin{array}{c}\Delta_{x} / F_{x} \\
\left(10^{-6} \mathrm{~m} / \mathrm{M}\right)\end{array}$ & $\begin{array}{c}\Delta_{y} / F_{y} \\
\left(10^{-3} \mathrm{~m} / \mathrm{M}\right)\end{array}$ & $\begin{array}{c}\Delta_{z} / F_{z} \\
\left(10^{-4} \mathrm{~m} / \mathrm{M}\right)\end{array}$ & $\begin{array}{c}\Delta_{z} / M_{y} \\
\left(10^{-2} / \mathrm{N}\right)\end{array}$ & $\begin{array}{c}\Delta_{y} / M_{z} \\
\left(10^{-1} / \mathrm{N}\right)\end{array}$ & $\begin{array}{c}\alpha_{x} / M_{x} \\
(\mathrm{rad} / \mathrm{N} \cdot \mathrm{m})\end{array}$ & $\begin{array}{c}\alpha_{y} / M_{y} \\
(\mathrm{rad} / \mathrm{N} \cdot \mathrm{m})\end{array}$ & $\begin{array}{c}\alpha_{z} / M_{z} \\
(\mathrm{rad} / \mathrm{N} \cdot \mathrm{m})\end{array}$ \\
\hline Theory & 3.0424 & 2.0864 & 1.5236 & -1.0639 & 1.6706 & 0.9623 & 0.8610 \\
Simulation & 3.0837 & 2.1845 & 1.5946 & -1.1915 & 1.6921 & 1.0690 & 0.9040 \\
Error (\%) & 1.3393 & 4.4907 & 4.4525 & 10.709 & 1.2706 & 9.9832 & 4.7590 \\
\hline
\end{tabular}

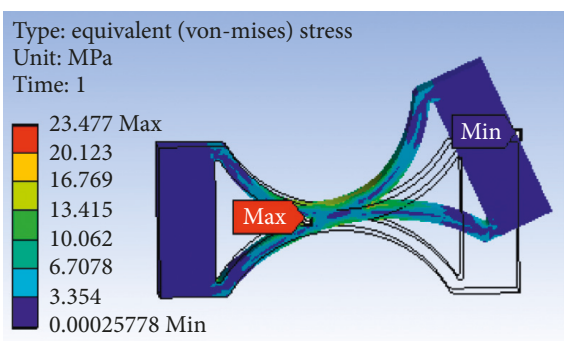

(a)

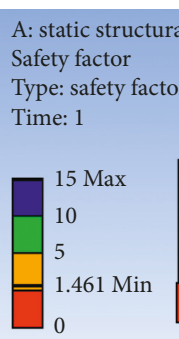

Safety factor

Type: safety factor

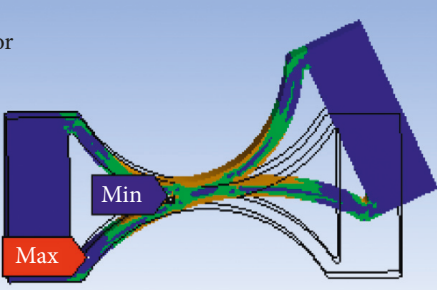

(b)

FIgURE 7: Simulation result of the X-shaped compliant joint at a rotation of $30^{\circ}$. (a) Stress diagram. (b) Safety factor.

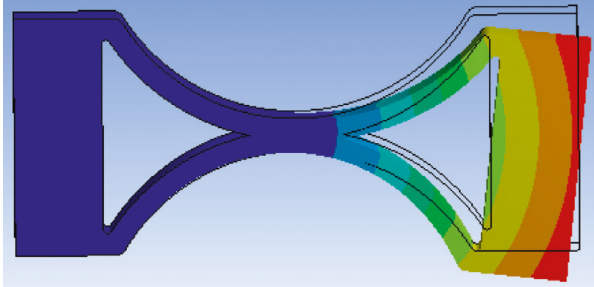

(a)

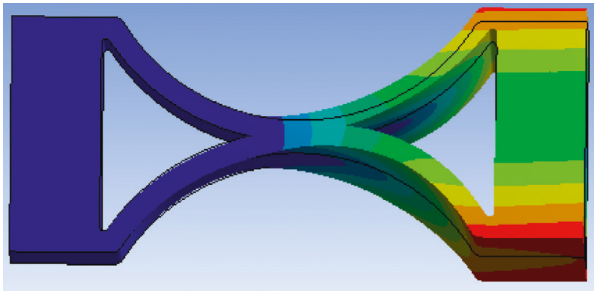

(c)

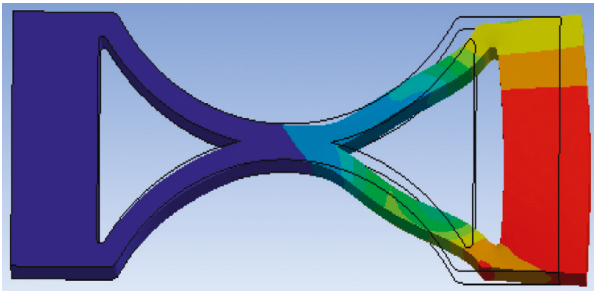

(e)

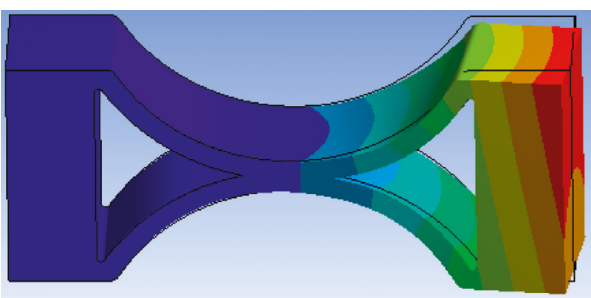

(b)

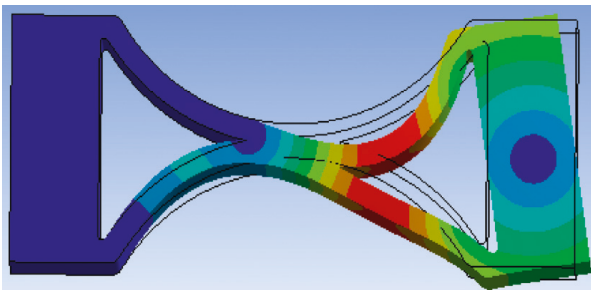

(d)

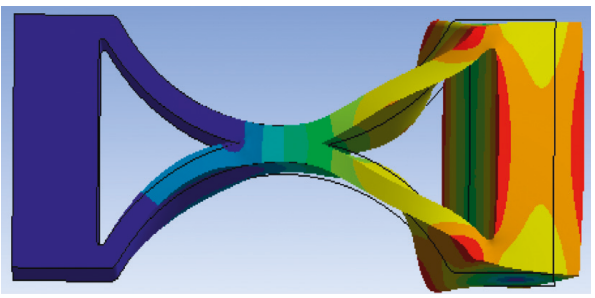

(f)

FIgURE 8: The first six-order mode shapes of X-shaped compliant joint. (a) $1^{\text {st }}$. (b) $2^{\text {nd }}$. (c) $3^{\text {rd }}$. (d) $4^{\text {th }}$. (e) $5^{\text {th }}$. (f) $6^{\text {th }}$.

TABLE 3: The first six-order natural frequency of the $\mathrm{X}$-shaped compliant joint.

\begin{tabular}{lcccccc}
\hline Mode & The first & The second & The third & The fourth & The fifth & The sixth \\
\hline Natural frequency $(\mathrm{Hz})$ & 315.6 & 1137.0 & 1511.1 & 3191.3 & 4302.9 & 5979.9 \\
\hline
\end{tabular}


From the first six-order natural frequency of the $\mathrm{X}$-shaped compliant joint, it can be seen that only the first order natural frequency is much smaller, and there is a big difference between the first order natural frequency and other orders' natural frequency. So, the X-shaped compliant joint has only one degree of freedom. From the mode diagram, we can find that the degree of freedom rotates around the $z$-axis.

In summary, $\mathrm{X}$-shaped compliant joint can be used to replace the revolute pair connecting the active rod and the driven rod in the 3-RRS SPM. By changing the material and size of the compliant joint, the compliance of the 3-RXS mechanism can be also adjusted to meet the needs of different angles, compliance and strength rehabilitation stage.

The model of the compliant joint can be established based on the $1 \mathrm{R}$ pseudo-rigid-body model (PRBM), as shown in Figure 9 [37]. Through the rotational compliance of the X-shaped compliant joint, the stiffness of the torsion spring in the pseudo-rigid model of the 3-RXS mechanism is determined, which plays a key role in building the overall compliance model of 3-RXS mechanism. Therefore, as shown in Figure 10, the $\mathrm{X}$-shaped compliant joint is regarded as the traditional joint with torsion spring whose rotational stiffness is $K_{z}$ :

$$
K_{z}=C_{z}^{-1}
$$

\section{The Design and Analysis of the 3-RXS Rigid-Flexible Parallel Mechanism for a Neck Brace}

Most neck braces are static or rigid, which causes movement impact in assisting the head's movement. As thus, a rigidflexible 3-RXS mechanism for a neck brace is developed. It utilizes elastic deformation of the X-shaped compliant joints to transmit force and absorb impact, which could make the neck brace more comfortable and safer during the rehabilitation exercise.

\subsection{The Design of the 3-RXS Rigid-Flexible Parallel} Mechanism for a Neck Brace. In the 3-RRS SPM illustrated in Figure 11, all of the three limbs are identical. The angle between the bottom joint and the middle joint and that between the middle joint and the top joint are both $90^{\circ}$. The orientation of the moving platform is expressed by $Z-Y-X$ Euler angles $(\alpha, \beta$, and $\gamma)$. Next, based on the rigid body replacement method, a new rigid-flexible parallel mechanism called the 3-RXS mechanism (Figure 12) is designed by using the X-shaped compliant joints (Figure 1) to replace the middle joints in the 3-RRS SPM (Figure 11).

As shown in Figure 13(a), the structure of the neck brace is designed preliminarily, which is composed of a base that rests on the shoulders, secured by a pair of straps, a headband worn around the forehead acting as an end-effector, and three rotational X-spherical (3-RXS) chains locating at the back of the head, left shoulder, and right shoulder symmetrically. The

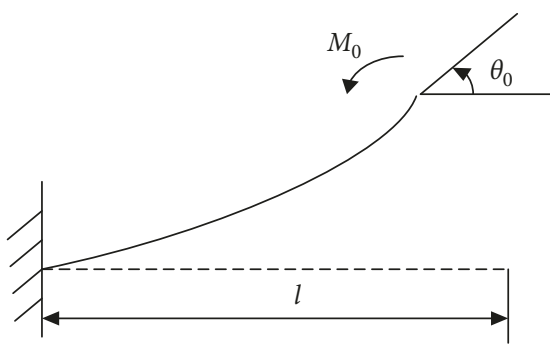

(a)

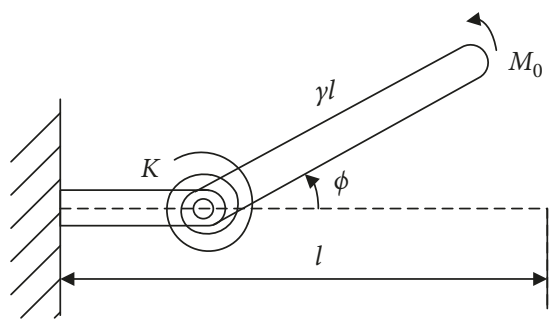

(b)

Figure 9: (a) Compliant beam. (b) Pseudo-rigid-body model.

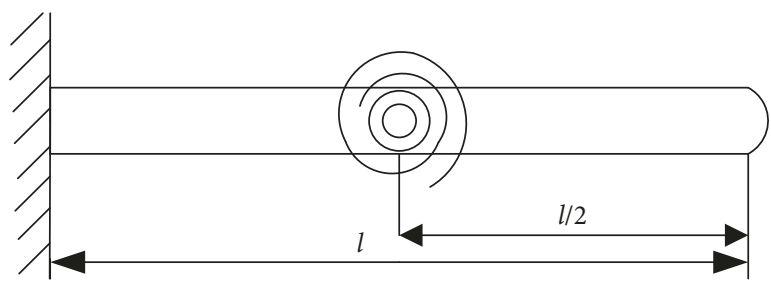

FIGURE 10: 1R pseudo-rigid body model of the X-shaped compliant joint.

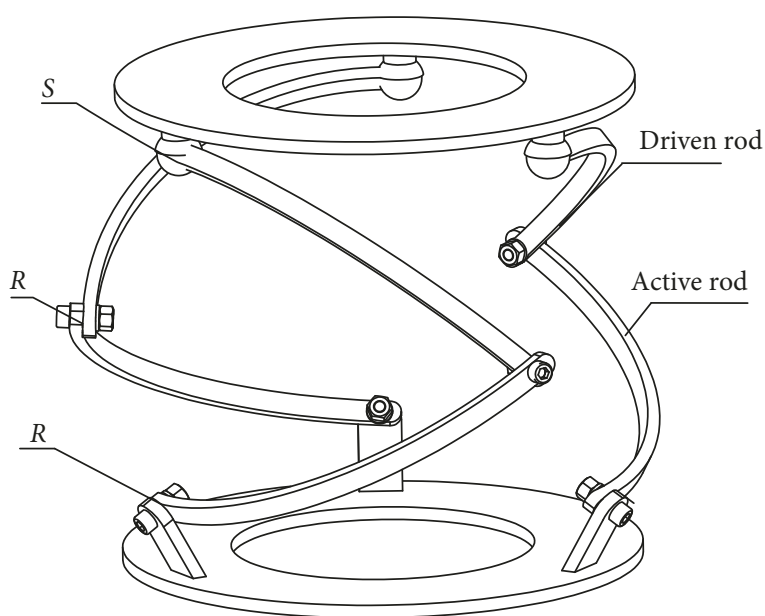

FIGURE 11: 3-RRS SPM.

numbers from 1 to 11 are the headband, the driven rod, the lift compliant joint, the right compliant joint, the behind compliant joint, the active rod, the decelerator, the motor, the base, head strap, and shoulder straps, respectively. From the 1R pseudo-rigid-body model of the X-shaped compliant joint, the 


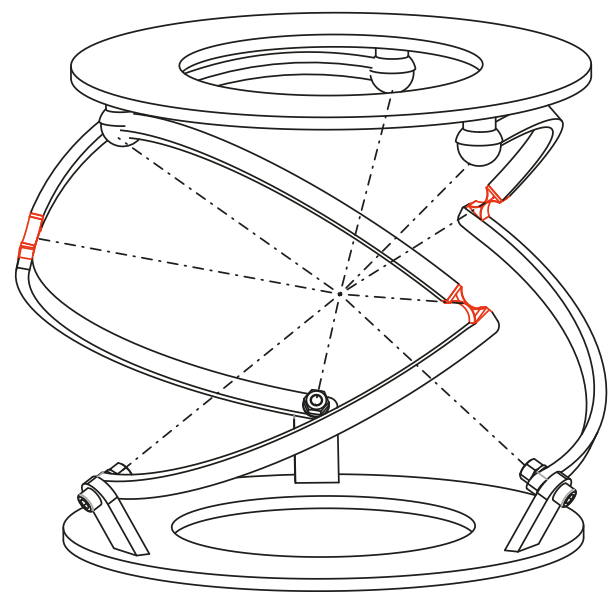

FIGURE 12: A 3-RXS mechanism.

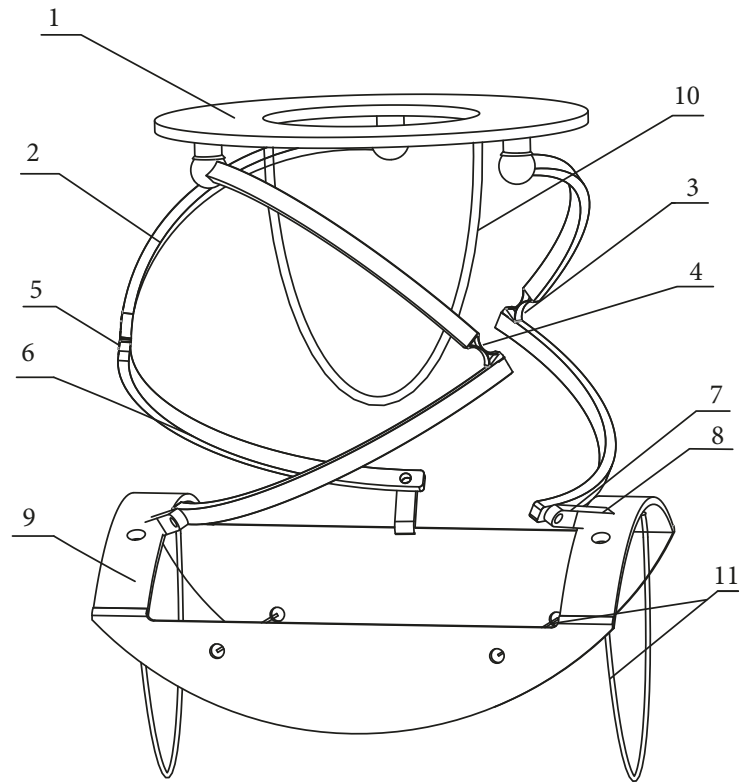

(a)

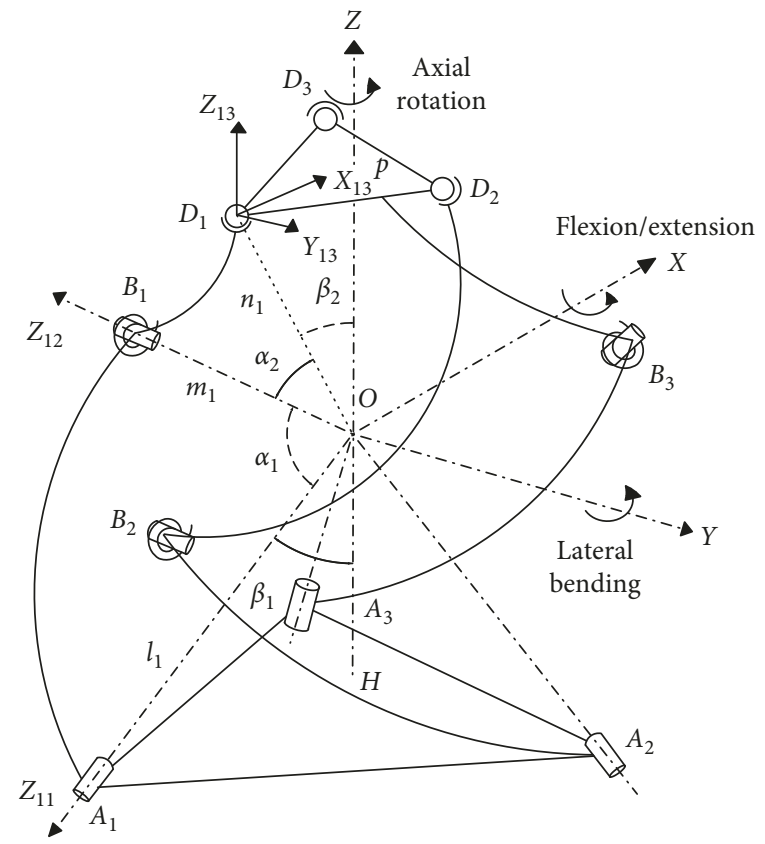

(b)

FIgURE 13: (a) The neck brace with compliant joints. (b) Pseudo-rigid-body model.

pseudo-rigid-body model of the 3-RXS mechanism can be obtained as shown in Figure 13(b).

4.2. The Rotation Workspace Analysis of the 3-RXS Rigid-Flexible Parallel Mechanism. The kinematics of the PRBM is independent of the stiffness of the torsion spring. Thus, the kinematics of the 3-RXS rigid-flexible parallel mechanism is the same as that of the 3-RRS SPM. The inverse displacement analysis of the 3-RRS SPM has been carried out $[24,25]$. To ensure the safety and obtain a neck brace with proper workspace, we focus on the analysis of rotation workspace. So, the results of the inverse displacement analysis are rewritten as shown in equations (34) (39).

As shown in Figure 13(b), the position of each joint axis is represented by unit vectors $l_{i}, m_{i}$, and $n_{i}$ in limb $i(i=1,2,3)$, respectively. The inverse displacement analysis is to solve three driving angles $\left(\varphi_{1}, \varphi_{2}\right.$, and $\left.\varphi_{3}\right)$ for a given orientation $X$ $Y$-Z Euler angles $(\alpha, \beta$, and $\gamma)$ of the moving platform:

$$
\begin{aligned}
& m_{i}=\left(\begin{array}{c}
m_{i x} \\
m_{i y} \\
m_{i z}
\end{array}\right)=\left(\begin{array}{c}
c \delta_{i} s \alpha_{1} s \theta_{1}+s \delta_{i} s \beta_{1} c \alpha_{1}-s \delta_{i} c \beta_{1} s \alpha_{1} c \theta_{1} \\
s \delta_{i} s \alpha_{1} s \theta_{1}-c \delta_{i} s \beta_{1} c \alpha_{1}+c \delta_{i} c \beta_{1} s \alpha_{1} c \theta_{1} \\
-c \beta_{1} c \alpha_{1}-s \beta_{1} s \alpha_{1} c \theta_{1}
\end{array}\right) \text {, } \\
& n_{i 0}=\left(s \delta_{i} s \beta_{2},-c \delta_{i} s \beta_{2}, c \beta_{2}\right)^{T} \text {, } \\
& R=\left(\begin{array}{ccc}
c \beta c \gamma & -c \beta s \gamma & s \beta \\
c \alpha s \gamma+c \gamma s \alpha s \beta & c \alpha c \gamma-s \alpha s \beta s \gamma & -c \beta s \alpha \\
s \alpha s \gamma-c \alpha c \gamma s \beta & c \gamma s \alpha+c \alpha s \beta s \gamma & c \alpha c \beta
\end{array}\right)
\end{aligned}
$$




$$
n_{i}=R n_{i 0}
$$

where $i=1,2,3$ and $\delta_{i}=0,2 \pi / 3,4 \pi / 3 . \alpha_{1}$ is the central angle of the curved link $A_{i} B_{i}, \alpha_{2}$ is the central angle of the curved link $B_{i} D_{i}, \beta_{1}$ is the cone angle of the base, and $\beta_{2}$ is the cone angle of the moving platform.

$$
\theta_{i}=2 \tan ^{-1}\left(x_{i}\right)=2 \tan ^{-1}\left(\frac{-A_{i} \pm \sqrt{A_{i}^{2}-C_{i}^{2}+B_{i}^{2}}}{2\left(C_{i}-B_{i}\right)}\right), \quad i=1,2,3
$$

where $A_{i}=c \delta_{i} s \alpha_{1} n_{i x}+s \delta_{i} s \alpha_{1} n_{i y}, B_{i}=-s \delta_{i} c \beta_{1} s \alpha_{1} n_{i x}+c \delta_{i} c \beta_{1}$ $s \alpha_{1} n_{i y}-s \beta_{1} s \alpha_{1} n_{i z}, C_{i}=s \delta_{i} s \beta_{1} c \alpha_{1} n_{i x}-c \delta_{i} s \beta_{1} c \alpha_{1} n_{i y}-c \beta_{1} c \alpha_{1}$ $n_{i z}-c \alpha_{2}$.

$$
\phi_{i}=\theta_{i} \pm\left|\theta_{0}\right|, \quad i=1,2,3,
$$

where $\theta_{i}(i=1,2,3)$ is the rotation angle of the motor, $\theta_{0}$ is the initial angle of the motor, and $\varphi_{i}$ is the input angle of the motor.

The 3-RXS mechanism has three rotational degrees of freedom. The rotation workspace is studied in the subsequent content.

A modified Euler angle shown in Figure 14 is used to describe the three-dimensional rotations of the mechanism. The rotation matrix in this case is

$$
\begin{aligned}
\mathbf{R}_{m}= & \mathbf{R}_{z}(\varphi) \mathbf{R}_{y}(\theta) \mathbf{R}_{z}(-\varphi) \mathbf{R}_{z}(\psi) \\
= & {\left[\begin{array}{ccc}
c \varphi c \theta c(\psi-\varphi)-s \varphi s(\psi-\varphi) & -c \varphi c \theta s(\psi-\varphi)-s \varphi c(\psi-\varphi) & c \varphi s \theta \\
s \varphi c \theta c(\psi-\varphi)+c \varphi c(\psi-\varphi) & -s \varphi c \theta s(\psi-\varphi)+c \varphi c(\psi-\varphi) & s \varphi s \theta \\
-s \theta c(\psi-\varphi) & s \theta s(\psi-\varphi) & c \theta
\end{array}\right], }
\end{aligned}
$$

where $s^{*}$ and $c^{*}$ are the $\sin \left({ }^{*}\right)$ and $\cos \left({ }^{*}\right)$, respectively.

The following two constraints are considered to influence the size of the workspace of the 3-RXS mechanism.

4.2.1. Angular Constraint of Kinematic Pairs. The constraint conditions of the kinematic pairs can be expressed as follows:

$$
\begin{gathered}
\phi_{\min } \leq \phi_{i}(i=1,2,3) \leq \phi_{\max } \\
\phi_{c \min } \leq \phi_{c i}(i=1,2,3) \leq \phi_{c \max }
\end{gathered}
$$

where $\varphi_{i}$ is the input angle of the active $\operatorname{rod}, \varphi_{\min }$ is the minimum input angle of the active rod, $\varphi_{\max }$ is the maximum input angle of the active $\operatorname{rod}, \varphi_{c i}$ is the rotation angle of the spherical pair, $\varphi_{c \min }$ is the minimum rotation angle of the spherical pair, and $\varphi_{c \max }$ is the maximum rotation angle of the spherical pair.

4.2.2. Interference Constraint of Rods. The rod constraints between different branches can be expressed as

$$
\begin{gathered}
D_{z i} \geq D, \\
D_{c i} \geq D, \\
D_{z c i} \geq D,
\end{gathered}
$$

$$
i=1,2,3 \text {, }
$$

where $D$ is the thickness of active rods and the passive rods, $D_{z i}$ is the shortest distance between active rods in adjacent branches, $D_{c i}$ is the shortest distance between passive rods in adjacent branches, and $D_{z c i}$ is the shortest distance between passive and active rods in adjacent branches.

The position of the center point $p$ of the moving platform in the fixed coordinate system is obtained as

$$
\left(X_{P}, Y_{P}, Z_{P}\right)^{T}=R_{m}\left[\begin{array}{c}
0 \\
0 \\
R_{1} \cos \beta_{2}
\end{array}\right]=\left[\begin{array}{c}
R_{1} c \phi s \theta c \beta_{2} \\
R_{1} s \phi s \theta c \beta_{2} \\
R_{1} c \theta c \beta_{2}
\end{array}\right],
$$

where $R_{1}$ is the spherical radius of the passive rod and the active rod.

According to equations (32) (42), the rotation workspace of the mechanism can be directly obtained. A cylindrical coordinate system is used to search the reachable workspace of the mechanism with geometric parameters $\alpha_{1}=\alpha_{2}=90^{\circ}, \beta_{1}=60^{\circ}$, and $\beta_{2}=45^{\circ}$, as shown in Figure 15.

From the workspace, we find that the 3-RXS mechanism for the neck brace can assist the neck to achieve flexion and extension, lateral bending, and axial torsion. The workspace is a part of spherical surface and has a large range.

4.3. The Kinematics Simulation of the 3-RXS Rigid-Flexible Parallel Mechanism. To verify the kinematics analysis and to show the feasibility of the 3-RXS mechanism for neck rehabilitation, the kinematics simulations of the 3-RXS mechanism and the 3-RRS mechanism have been carried out and compared with the theoretic kinematics as well.

The rotation range of the neck is from $-45^{\circ}$ to $45^{\circ}$, and the motion range of the neck brace can be initially set from 


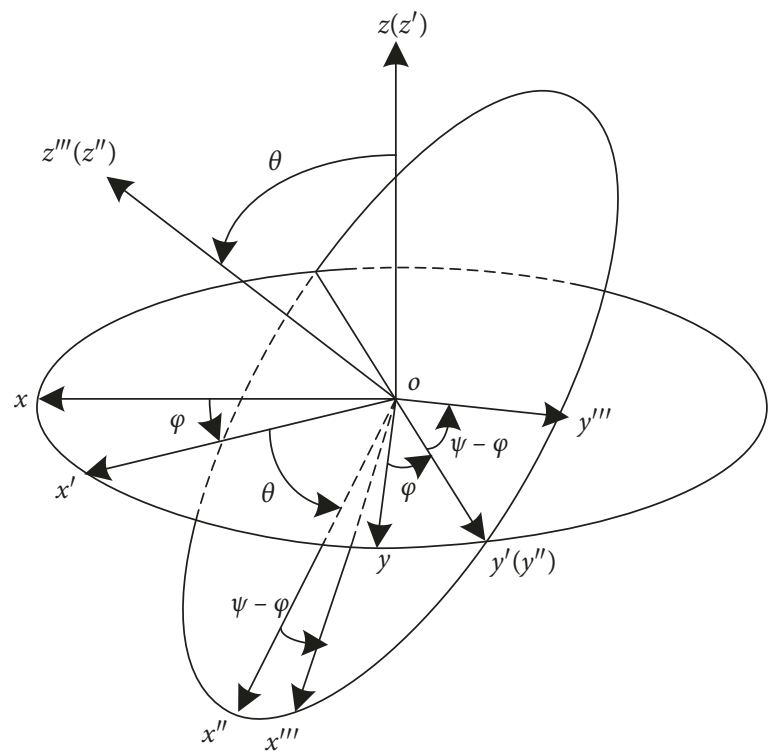

Figure 14: Modified Euler angles.

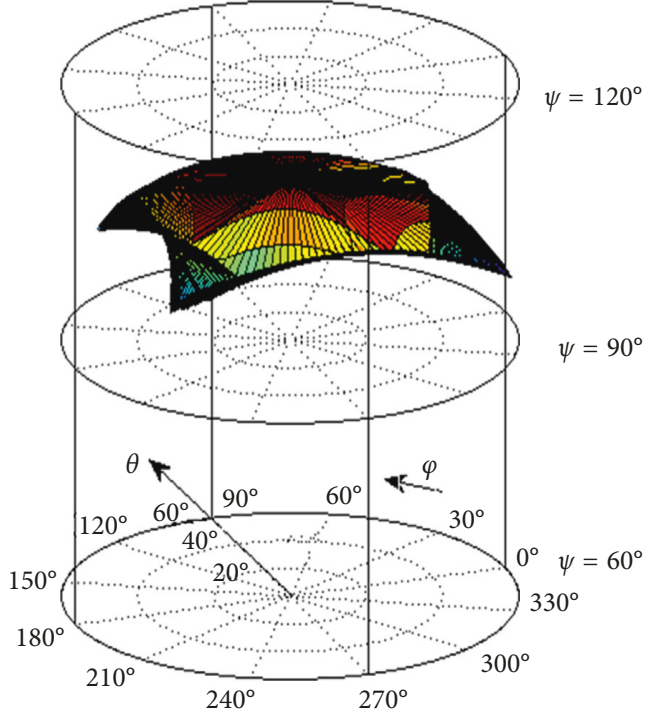

(a)

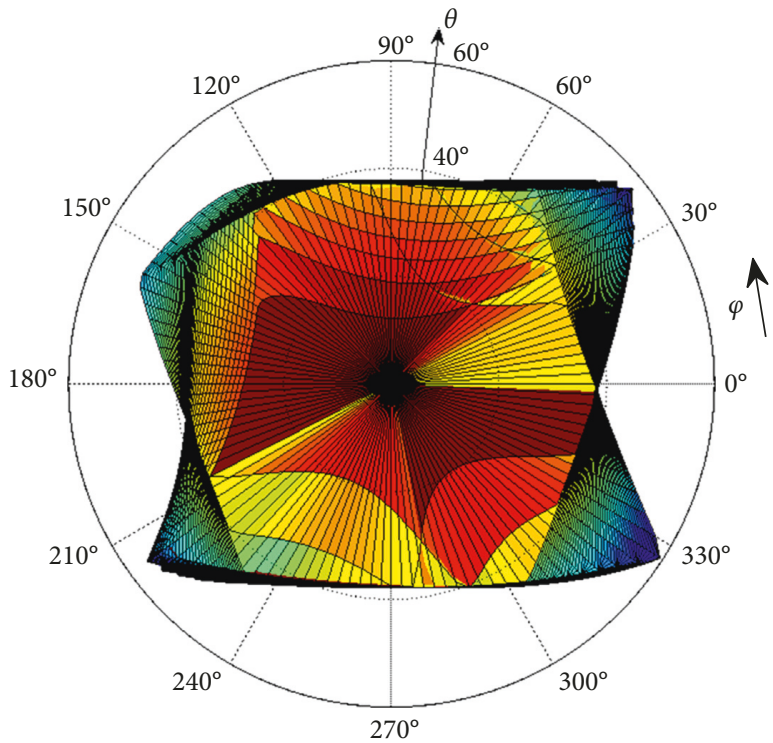

(b)

FIGURE 15: Rotation workspace of the 3-RXS mechanism. (a) 3D view. (b) Vertical view.

$-30^{\circ}$ to $30^{\circ}$ to avoid excessive exercise [38]. According to equations (34) (39) of the 3-RRS SPM, the three rotation angles of drive motors can be, respectively, obtained when the mechanism assists the patient to achieve the flexion and extension, lateral bend, and torsion. The same drivers are applied to the 3-RXS mechanism for kinematics simulation. The simulation rotation curves of driving motors in the 3-RXS mechanism and 3-RRS mechanism changing with time are shown in magenta dotted lines and blue dotted lines, respectively. The theoretic curves of kinematics analysis are shown in red solid lines, as shown in Figure 16.
It can be found that during the flexion and extension, lateral bend, and torsion, the rotation curves of the 3-RRS mechanism obtained by simulations basically coincide with that of theoretic analysis. The angle derivation appears between the theoretic and simulation curves of the 3-RXS mechanism due to the affection of gravity and simplification of the pseudo-rigid-body model.

As shown in Figure 16, the 3-RXS mechanism can rotate about $-19^{\circ}$ to $+17^{\circ}$ during the flexion and extension, $-15^{\circ}$ to $+20^{\circ}$ during the lateral bend and $-32^{\circ}$ to $+25^{\circ}$ during the torsion. The ranges of rotations of the 3 -RXS mechanism can basically meet the requirements of the neck rehabilitation 


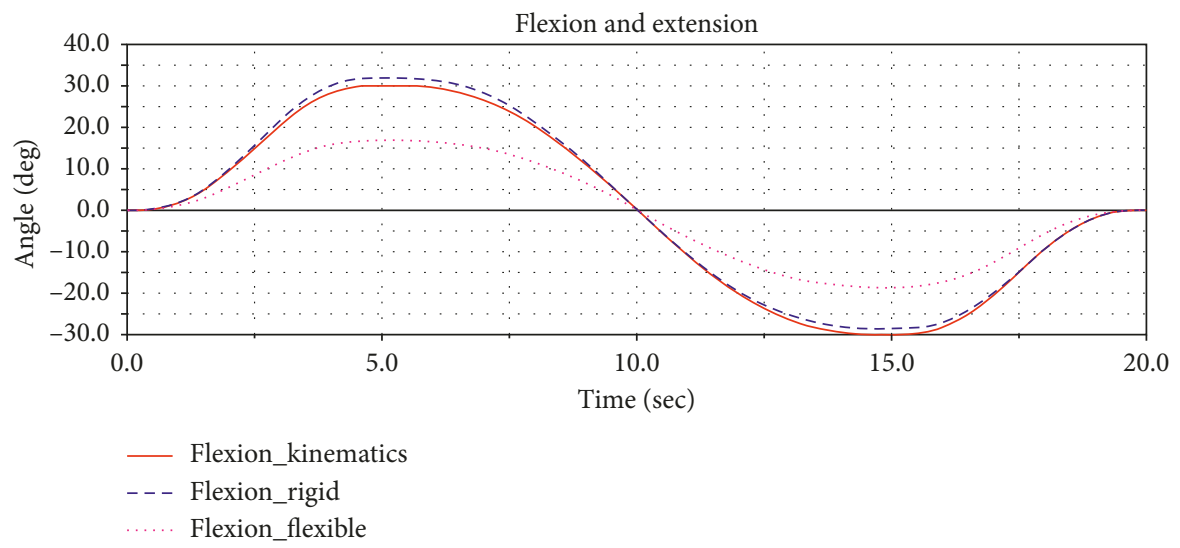

(a)

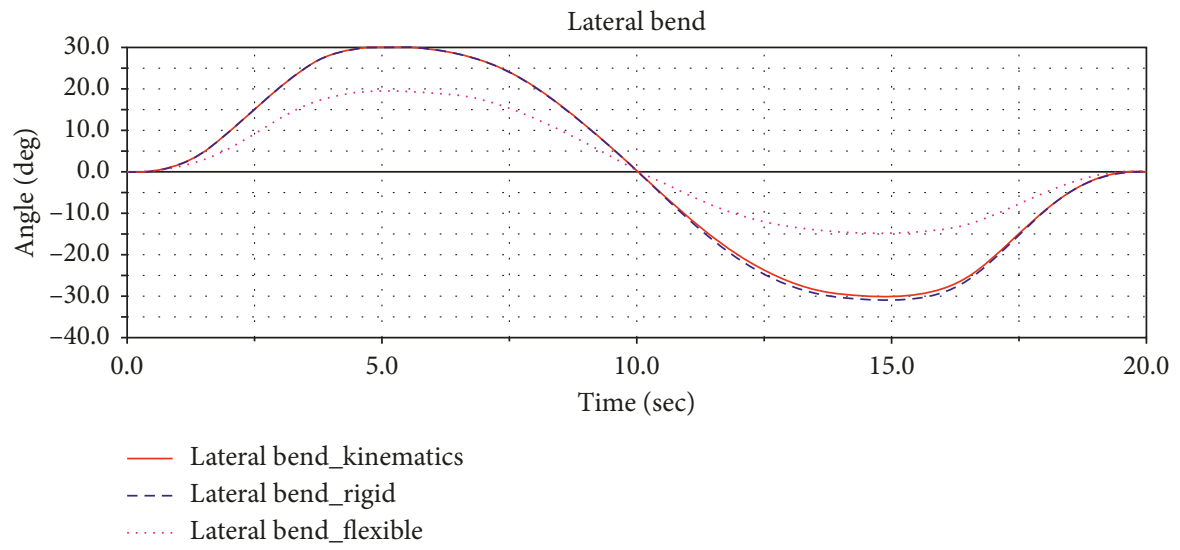

(b)

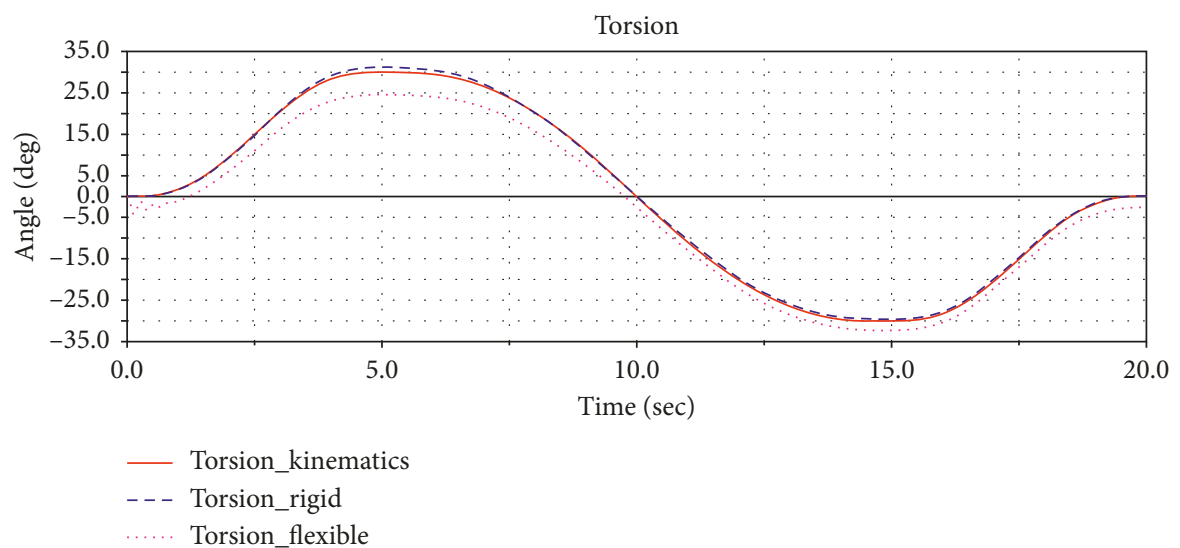

(c)

Figure 16: The rotation angles $\alpha, \beta$, and $\gamma$ of the moving platform changing with time $t$ during flexion and extension, lateral bend, and torsion. (a) The rotation angle $\alpha$ changes with time $t$ during flexion and extension. (b) The rotation angle $\beta$ changes with time $t$ during lateral bend. (c) The rotation angle $\gamma$ changes with time $t$ during torsion.

exercise, which also proves the feasibility of the 3-RXS mechanism for the neck brace.

Besides, because of the existence of the X-shaped compliant joint, the 3-RXS mechanism generates vibration in start-up moment. The above deformation and vibration characteristics are reflected in the movement of the compliant joint, which is the unique property of the compliant part. The deformation can absorb the impact during the rehabilitation process and make the rehabilitation exercise more comfortable. The vibration may be reduced from the aspects of structural design, material selection, drive selection, and so on. 


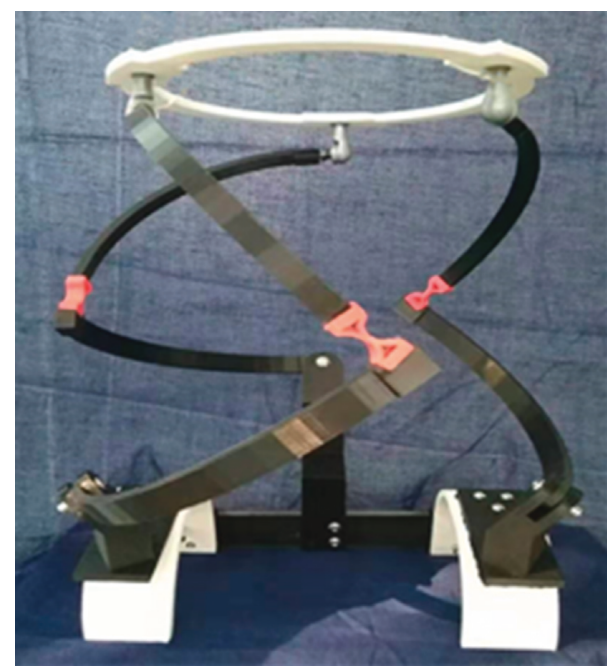

FIGURE 17: 3-RXS mechanism prototype.

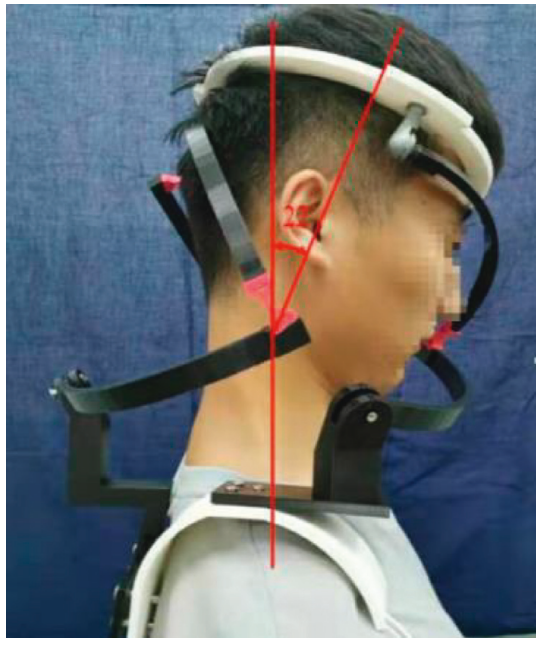

(a)

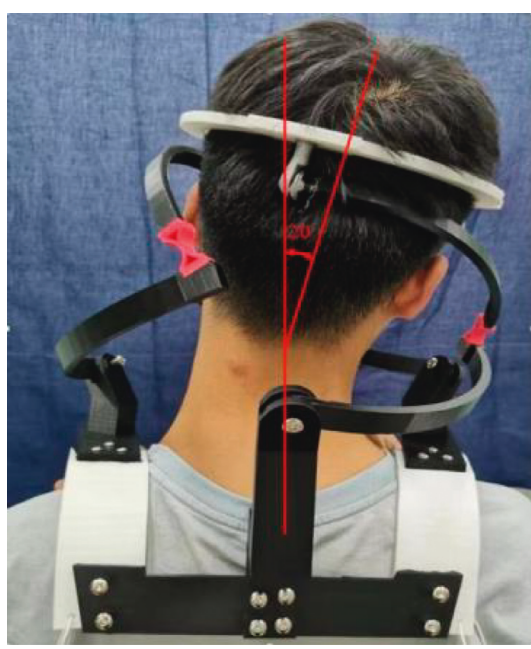

(d)

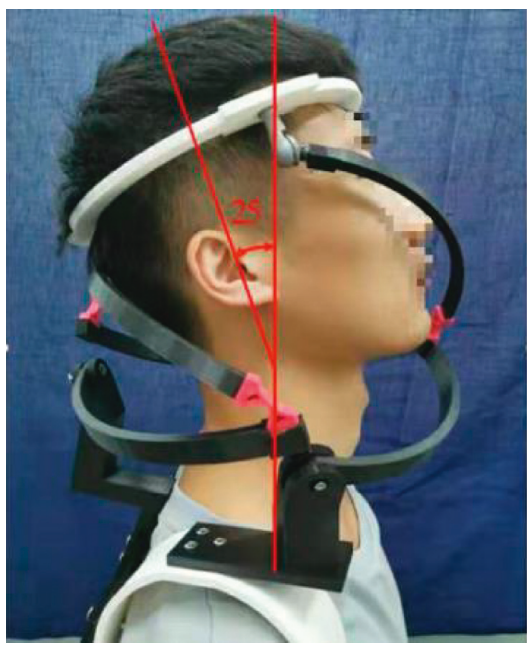

(b)

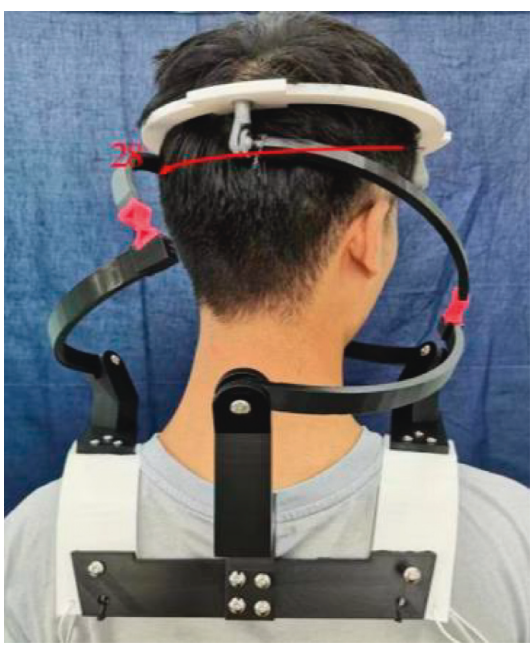

(e)

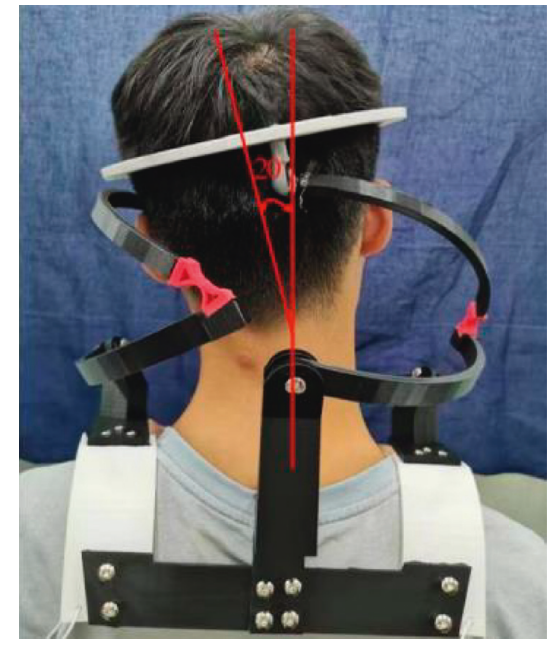

(c)

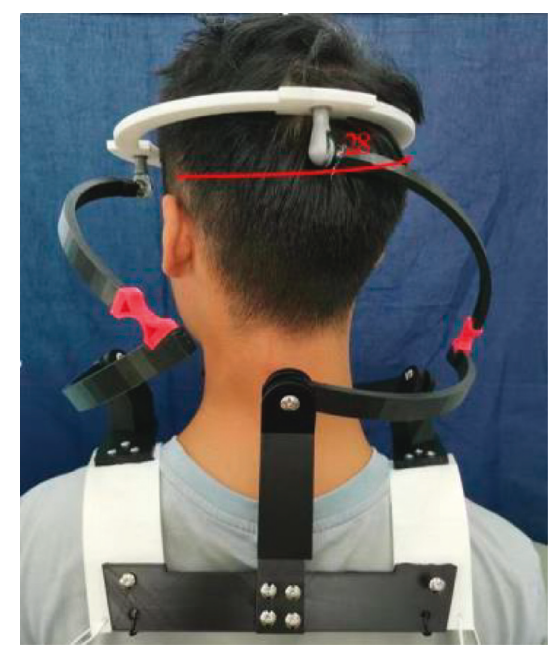

(f)

Figure 18: Postures of the 3-RXS mechanism prototype. (a) Flexion. (b) Extension. (c) Left bend. (d) Right bend. (e) Left torsion. (f) Right torsion. 


\section{Effect of the 3-RXS Mechanism Prototype as a Neck Brace}

In order to intuitively reflect the main function of the 3-RXS mechanism as a neck brace, a prototype corresponding to Figure 13(a) has been made, as shown in Figure 17. In addition, six postures are given in which the human body wears the mechanism prototype, and the rotation angles are simply measured, as shown in Figure 18.

From the example measurements in Figure 18, the maximum rotation angles of the 3 -RXS mechanism are about flexion $22^{\circ}$ and extension $25^{\circ}$, lateral bend $20^{\circ}$, and torsion $28^{\circ}$, which indicates that the 3 -RXS mechanism can basically meet the requirements of the neck rehabilitation exercise. In addition, by virtue of restoring deformation energy, the 3-RXS mechanism can be applied to not only passive rehabilitation training but also active self-adaptive training.

At present, this research work only builds a simple 3RXS mechanism prototype for neck rehabilitation mechanism. And, we carry out a simple measurement of the rotation angles, which initially reveals that the 3-RXS mechanism can be applied to neck rehabilitation. The further research studies such as dynamics analysis, prototype construction, and experimental testing are underway.

\section{Conclusions}

A rigid-flexible parallel mechanism called the 3-RXS mechanism as a neck brace is presented. The spatial compliance matrix, axis drift, and DoF of an X-shaped compliant joint are deduced, where the six-dimensional compliance matrix is derived by solving the quarter compliant joint with the microintegral method firstly and then connecting it in series and parallel. It is concluded that the $\mathrm{X}$-shaped compliant joint can be used to replace the revolute pair in the 3-RRS SPM and then a 3-RXS rigid-flexible parallel mechanism is obtained.

Rotation workspace of the 3-RXS mechanism is analyzed, which indicates that the 3-RXS mechanism can assist the neck to achieve flexion and extension, lateral bend, and axial torsion. By the comparison of rotation curves from theoretic analysis and simulation of the 3-RXS mechanism, it is shown that the motion trends are same, and the 3-RXS mechanism can rotate from $-19^{\circ}$ to $+17^{\circ}$ during the flexion and extension, from $-15^{\circ}$ to $+20^{\circ}$ during the lateral bend, and from $-32^{\circ}$ to $+25^{\circ}$ during the torsion, which basically meets the needs of neck rehabilitation.

A simple 3-RXS mechanism prototype is made, and a primary rotation experiment for angle measurement is performed, which preliminarily shows the effect of the 3RXS mechanism for a neck brace.

The further research studies such as dynamics analysis, prototype construction, and experimental testing will be considered in our future work.

\section{Appendix}

$$
\begin{aligned}
C_{55} & =\frac{\alpha_{y}}{M_{y}}=\frac{12 r}{E b^{3}} \int_{-\lambda_{m}}^{0} \frac{\cos \lambda}{\sqrt{R^{2}-(r \sin \lambda)^{2}}-r \cos \lambda} d \lambda \\
& =\frac{12 r}{E b^{3}\left(R^{2}-r^{2}\right)} N_{55}, \\
C_{35} & =\frac{\alpha_{y}}{F_{z}}=\frac{12 r^{2}}{E b^{3}} \int_{-\lambda_{m}}^{0} \frac{\cos \lambda \sin \lambda}{\sqrt{R^{2}-(r \sin \lambda)^{2}}-r \cos \lambda} d \lambda \\
& =\frac{12 r^{2}}{E b^{3}\left(R^{2}-r^{2}\right)} N_{35}, \\
C_{44}=\frac{\alpha_{x}}{M_{x}} & =\int_{-\lambda_{m}}^{0} \frac{12 r \cos \lambda}{G b\left(R^{2}-r^{2}+b^{2}\right)\left(\sqrt{R^{2}-(r \sin \lambda)^{2}}-r \cos \lambda\right)} d \lambda \\
& =\frac{12 r}{G b\left(R^{2}-r^{2}+b^{2}\right)\left(R^{2}-r^{2}\right)} N_{44}, \\
C_{11}=\frac{\Delta_{x}}{F_{x}} & =\int_{-\lambda_{m}}^{0} \frac{r \cos \lambda}{E b\left(\sqrt{R^{2}-(r \sin \lambda)^{2}}-r \cos \lambda\right)} d \lambda \\
& =\frac{r}{E b\left(R^{2}-r^{2}\right)} N_{11},
\end{aligned}
$$




$$
\begin{aligned}
& C_{62}=\frac{\Delta y}{M_{z}}=-\int_{-\lambda_{m}}^{0} \frac{12 r^{2} \cos \lambda \sin \lambda}{E b\left(\sqrt{R^{2}-(r \sin \lambda)^{2}}-r \cos \lambda\right)^{3}} d \lambda \\
& =\frac{-12 r^{2}}{E b\left(R^{2}-r^{2}\right)^{3}} N_{62} \\
& C_{22}=\frac{12 r^{3} N_{22}}{E b\left(R^{2}-r^{2}\right)^{3}}+\frac{k r N_{55}}{G b\left(R^{2}-r^{2}\right)}, \\
& C_{53}=\frac{\Delta z}{M_{y}}=\int_{-\lambda_{m}}^{0} \frac{12 r^{2} \cos \lambda \sin \lambda}{E b^{3}\left(\sqrt{R^{2}-(r \sin \lambda)^{2}}-r \cos \lambda\right)} d \lambda \\
& =\frac{12 r^{2}}{E b^{3}\left(R^{2}-r^{2}\right)} N_{53} \text {, } \\
& C_{33}=\frac{12 r^{3} N_{33}}{E b^{3}\left(R^{2}-r^{2}\right)}+\frac{k r N_{55}}{G b\left(R^{2}-r^{2}\right)}, \\
& N_{66}=\left[\left(R^{2}-r^{2} \sin ^{2} \lambda_{m}\right)^{3 / 2} \sin \lambda_{m}+\frac{3 R^{4}}{32 r}\left(4 \lambda_{m}-\sin 4 \lambda_{m}\right)\right]+\frac{3 r}{2}\left[\left(R^{2}-\frac{r^{2}}{4}\right) \lambda_{m}+\frac{R^{2}}{2} \sin 2 \lambda_{m}+\frac{r^{2}}{16} \sin 4 \lambda_{m}\right] \\
& +\frac{3 R^{2} r}{2}\left[\left(1-\frac{R^{2}}{4 r^{2}}\right) \lambda_{m}+\left(\frac{1}{2}+\frac{R^{2}}{16 r^{2}}\right) \sin 2 \lambda_{m}\right]+r^{3}\left[\cos ^{3} \lambda_{m} \sin \lambda_{m}+\frac{3}{32}\left(4 \lambda_{m}-\sin 4 \lambda_{m}\right)\right] \\
& N_{26}=\frac{1}{5 r^{2}}\left[\left(R^{2}-r^{2} \sin ^{2} \lambda_{m}\right)^{5 / 2}-R^{5}\right]+\frac{r^{3}}{5}\left[\left(\cos ^{5} \lambda_{m}-1\right)\right] \\
& +\frac{1}{5}\left[5 R^{2} r\left(\cos ^{3} \lambda_{m}-1\right)+5 r^{3}\left[\left(1-\cos ^{3} \lambda_{m}\right)+3\left(\cos ^{5} \lambda_{m}-1\right)\right]\right] \\
& +\left[\left(\frac{R^{2}}{r^{2}}-1\right)\left(R^{3}-\left(R^{2}-r^{2} \sin ^{2} \lambda_{m}\right)^{3 / 2}\right)-\frac{3}{5 r^{2}}\left(R^{5}+\left(R^{2}-r^{2} \sin ^{2} \lambda_{m}\right)^{5 / 2}\right)\right] \\
& N_{55}=\frac{R^{2}}{2 r}\left(\arcsin \left(\frac{r \sin \lambda_{m}}{R}\right)+\frac{r \sin \lambda_{m}}{R^{2}}\left(R^{2}-r^{2} \sin ^{2} \lambda_{m}\right)^{1 / 2}+\frac{r^{2}}{R^{2}} \lambda_{m}+\frac{r^{2}}{2 R^{2}} \sin 2 \lambda_{m}\right), \\
& N_{22}=\frac{r}{24 R^{4}}\left[\left(1-4 R^{2}+4 r^{2} \sin ^{2} \lambda_{m}\right)\left(R^{2}-r^{2} \sin ^{2} \lambda_{m}\right)^{3 / 2} \sin \lambda_{m}\right] \\
& +\frac{r^{3}}{6}\left[\frac{1}{4} \cos ^{3} \lambda_{m} \sin \lambda_{m}+\frac{3}{8} \lambda_{m}+\frac{3}{16} \sin 2 \lambda_{m}-\cos ^{5} \lambda_{m} \sin \lambda_{m}\right] \\
& +\frac{3 r}{16}\left[-\frac{8 r^{2}}{3} \sin ^{5} \lambda_{m} \cos \lambda_{m}+4\left(\frac{r^{2}}{6}+R^{2}\right) \sin ^{3} \lambda_{m} \cos \lambda_{m}-2 \lambda_{m}\left(\frac{r^{2}}{2}-R^{2}\right)+\left(\frac{r^{2}}{2}-R^{2}\right) \sin 2 \lambda_{m}\right] \\
& +\frac{1}{4 r^{2}}\left[\left(R^{2}-r^{2} \sin ^{2} \lambda_{m}\right)^{1 / 2} \sin \lambda_{m}\left[-2 r^{4} \sin ^{4} \lambda_{m}+\frac{R^{2} r^{2}}{2} \sin ^{2} \lambda_{m}+\frac{4 r^{3}}{R^{4}}\left(R^{2}-r^{2} \sin ^{2} \lambda_{m}\right)-\frac{2 r^{3}+R^{2} r}{R^{2}}\right]\right. \\
& \left.+\left(R^{2}-2 r^{2}\right) \arcsin \left(\frac{r}{R} \sin \lambda_{m}\right)\right] \\
& N_{35}=\frac{1}{3 r^{2}}\left[\left(R^{2}-r^{2} \sin ^{2} \lambda_{m}\right)^{3 / 2}-R^{3}+r^{3}\left(\cos ^{3} \lambda_{m}-1\right)\right] \text {, } \\
& N_{33}=\frac{1}{8 r^{2}}\left[\frac{R^{4}}{r} \arcsin \left(\frac{r}{R} \sin \lambda_{m}\right)-\sin \lambda_{m}\left(R^{2}-2 r^{2} \sin ^{2} \lambda_{m}\right)\left(R^{2}-r^{2} \sin ^{2} \lambda_{m}\right)^{1 / 2}\right] \\
& +\frac{r}{16}\left[\left(2 \lambda_{m}-\sin 2 \lambda_{m}+4 \sin ^{3} \lambda_{m} \cos \lambda_{m}\right)\right] \text {. }
\end{aligned}
$$




\section{Data Availability}

The data used to support the findings of this study are included within the article.

\section{Conflicts of Interest}

The authors declare that there are no conflicts of interest regarding the publication of this paper.

\section{Acknowledgments}

This work was supported by the National Science Foundation of China (no. 51975007) and Innovative Design and Research Methodology Project (no. 2H001014201702).

\section{References}

[1] T. G. Petheram, P. G. Hourigan, I. M. Emran, and C. R. Weatherley, "Dropped head syndrome: a case series and literature review," Spine, vol. 33, no. 1, pp. 47-51, 2008.

[2] P. Glazener, "Pilot study to determine the effectiveness of a new neck brace design for patients with amyotrophic lateral sclerosis," Journal of Nursing Education and Practice, vol. 4, no. 6, pp. 1-5, 2014.

[3] A. L. Ward, M. Sanjak, K. Duffy, and B. R. Brooks, "Power wheelchair prescription, utilization, satisfaction, and cost for patients with amyotrophic lateral sclerosis: preliminary data for evidence-based guidelines," Archives of Physical Medicine and Rehabilitation, vol. 91, no. 2, pp. 268-272, 2010.

[4] D. S. Wu, L. Wang, and P. Li, "A 6-DOF exoskeleton for head and neck motion assist with parallel manipulator and sEMG based control," in Proceedings of the 2016 International Conference on Control, Decision and Information Technologies (CoDIT), pp. 341-344, Saint Julian's, Malta, April 2016.

[5] H. Zhang, K. Albee, and S. K. Agrawal, "A spring-loaded compliant neck brace with adjustable supports," Mechanism and Machine Theory, vol. 125, pp. 34-44, 2018.

[6] H. H. Zhang and S. K. Agrawal, "Kinematic design of a dynamic brace for measurement of head/neck motion," IEEE Robotics and Automation Letters, vol. 2, no. 3, pp. 1428-1435, 2017.

[7] H. H. Zhang and S. K. Agrawal, "An active neck brace controlled by a joystick to assist head motion," IEEE Robotics and Automation Letters, vol. 3, no. 1, pp. 37-43, 2018.

[8] L. L. Howell, "Compliant mechanisms," in Encyclopedia of Nanotechnology, vol. 10, pp. 457-463, John Wiley \& Sons, Inc., Hoboken, NJ, USA, 2001.

[9] U. Bhagat, B. Shirinzadeh, L. Clark et al., "Design and analysis of a novel flexure-based 3-DOF mechanism," Mechanism and Machine Theory, vol. 74, pp. 173-187, 2014.

[10] M. Ling, J. Cao, L. L. Howell et al., "Kinetostatic modeling of complex compliant mechanisms with serial-parallel substructures: a semi-analytical matrix displacement method," Mechanism and Machine Theory, vol. 125, pp. 169-184, 2018.

[11] T. L. Wu, J. H. Chen, and S. H. Chang, "A six-DOF prismaticspherical-spherical parallel compliant nanopositioner," IEEE Transactions on Ultrasonics Ferroelectrics \& Frequency Control, vol. 55, no. 12, pp. 341-344, 2008.

[12] D. C. Handley, Y. K. Yong, and C. W. J. Zhang, "A simple and efficient dynamic modeling method for compliant micropositioning mechanisms using flexure hinges," in Proceedings of the Device \& Process Technologies for Mems Microelectronics \& Photonics III, vol. 5276, Perth, Western Australia, April 2004.

[13] H. H. Pham and I. M. Chen, "Stiffness modeling of flexure parallel mechanism," Precision Engineering, vol. 29, no. 4, pp. 467-478, 2005.

[14] J. J. Yu, D. F. Lu, and G. B. Hao, "Design and analysis of a 2DOF compliant parallel pan-tilt platform," Meccanica, vol. 51, no. 7, pp. 1559-1570, 2016.

[15] Y. Li and Q. Xu, "Design and analysis of a totally decoupled flexure-based XY parallel micromanipulator," IEEE Transactions on Robotics, vol. 25, no. 3, pp. 645-657, 2009.

[16] Y. Dong, F. Gao, and Y. Yue, "Modeling and experimental study of a novel 3-RPR parallel micro-manipulator," Robotics and Computer-Integrated Manufacturing, vol. 37, pp. 115124, 2016.

[17] H. Tang and Y. M. Li, "Design, analysis, and test of a novel 2-DOF nanopositioning system driven by dual mode," IEEE Transactions on Robotics, vol. 29, no. 3, pp. 650-662, 2013.

[18] Y. M. Li and Q. S. Xu, "A novel piezoactuated XY stage with parallel, decoupled, and stacked flexure structure for micro-/ nanopositioning," IEEE Transactions on Industrial Electronics, vol. 58, no. 8, pp. 3601-3615, 2011.

[19] L. L. Howell and A. A. Midha, "Parametric deflection approximations for end-loaded, large-deflection beams in compliant mechanisms," Journal of Mechanical Design, Transaction of the ASME, vol. 117, no. 1, pp. 156-165, 1995.

[20] J. B. Hopkins and M. L. Culpepper, "Synthesis of multi-degree of freedom, parallel flexure system concepts via freedom and constraint topology (FACT)-part I: principles," Precision Engineering, vol. 34, no. 2, pp. 259-270, 2010.

[21] P. V. Hull and S. Canfield, "Optimal synthesis of compliant mechanisms using subdivision and commercial FEA," Journal of Mechanical Design, vol. 128, no. 2, pp. 337-348, 2006.

[22] P. Bernardoni, P. Bidaud, C. Bidard et al., "A new compliant mechanisms design methodology based on flexible building blocks," in Proceedings of the Smart Structures \& Materials: Modeling, Signal Processing, \& Control, March 2004.

[23] C. M. Gosselin and J. F. Hamel, "The agile eye: a high-performance three-degree-of-freedom camera-orienting device," in Proceedings of the 1994 IEEE International Conference on Robotics and Automation, Nagoya, Japan, May 1994.

[24] S. Bai, M. R. Hansen, and J. Angeles, "A robust forwarddisplacement analysis of spherical parallel robots," Mechanism \& Machine Theory, vol. 44, no. 12, pp. 2204-2216, 2009.

[25] Z. Tao and Q. An, "Interference analysis and workspace optimization of 3-RRR spherical parallel mechanism," Mechanism and Machine Theory, vol. 69, no. 6, pp. 62-72, 2013.

[26] Y. T. Du, R. Q. Li, D. H. Li, and S. P. Bai, "An ankle rehabilitation robot based on 3-RRS spherical parallel mechanism," Advances in Mechanical Engineering, vol. 9, no. 8,Article ID 168781401771811,, 2017.

[27] J. F. Li, D. S. Li, B. W. Shen, and C. Z. Zhang, "Kinematical performance analysis of a 3-RRS spherical parallel mechanism for ankle rehabilitation," in Proceedings of the 2015 International Conference on Mechanical Engineering and Automation (ICMEA 2015), pp. 73-80, Phuket, Thailand, July 2015.

[28] Y. Tian, B. Shirinzadeh, and D. Zhang, "Closed-form compliance equations of filleted V-shaped flexure hinges for compliant mechanism design," Precision Engineering, vol. 34, no. 3, pp. 408-418, 2010. 
[29] M. Liu, X. Zhang, and S. Fatikow, "Design and analysis of a multi-notched flexure hinge for compliant mechanisms," Precision Engineering, vol. 48, pp. 292-304, 2017.

[30] R. Mutlu, G. Alici, M. Panhuis, and G. Spinks, "Effect of flexure hinge type on a $3 \mathrm{D}$ printed fully compliant prosthetic finger," in Proceedings of the 2015 IEEE International Conference on Advanced Intelligent Mechatronics (AIM), Busan, South Korea, July 2015.

[31] G. Chen, Y. Ma, and J. Li, "A tensural displacement amplifier employing elliptic-arc flexure hinges," Sensors and Actuators A: Physical, vol. 247, pp. 307-315, 2016.

[32] Z. Xie, L. Qiu, and D. Yang, "Design and analysis of outsidedeployed lamina emergent joint (OD-LEJ)," Mechanism and Machine Theory, vol. 114, pp. 111-124, 2017.

[33] M. Yang, Z. J. Du, Y. Chen, L. N. Sun, and W. Dong, "Static modelling and analysis of cross-spring flexure pivots with variable cross-section," Journal of Mechanical Engineering, vol. 54, no. 13, pp. 73-78, 2018.

[34] N. F. Wang, Z. Y. Zhang, X. M. Zhang, and X. H. Liang, "Performance comparison and analysis of three 2-DOF compliant precision positioning stages," Journal of Mechanical Engineering, vol. 54, no. 13, pp. 102-109, 2018.

[35] Y. Q. Yu and Q. Q. Li, "On the design, manufacturing and experiment of a new type flexural joint," Journal of Mechanical Engineering, vol. 54, no. 13, pp. 79-85, 2018.

[36] J. J. Yu, S. S. Bi, X. Yan, H. Z. Zhao, and G. H. Zong, Flexure Design: Analysis and Synthesis of Compliant Mechanism, Higher Education Press, Beijing, China, 2018.

[37] L. L. Howell, A. Midha, and T. W. Norton, "Evaluation of equivalent spring stiffness for use in a pseudo rigid body model of large deflection compliant mechanisms," Journal of Mechanical Design, vol. 118, no. 1, pp. 126-140, 1996.

[38] D. M. Gourie, A. Nalini, and S. Sandhya, "Early or late appearance of "dropped head syndrome" in amyotrophic lateral sclerosis," Journal of Neurology, Neurosurgery \& Psychiatry, vol. 74, no. 5, pp. 683-686, 2003. 


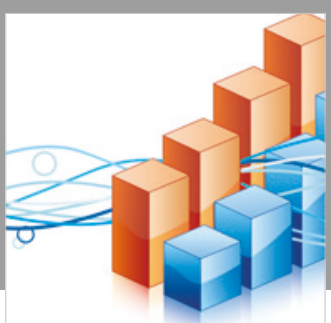

Advances in

Operations Research

\section{-n-m}
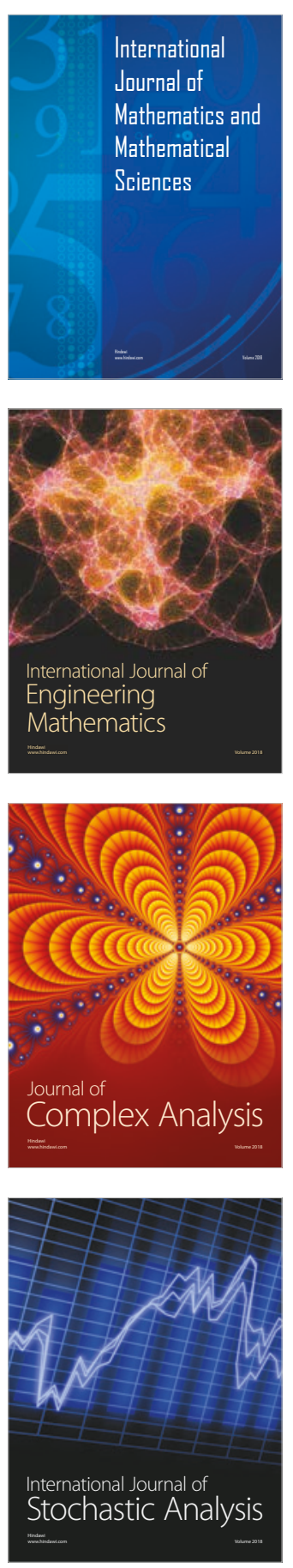
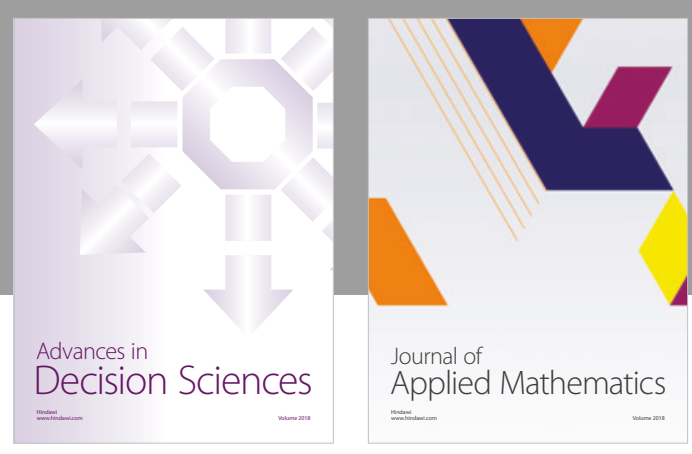

Journal of

Applied Mathematics
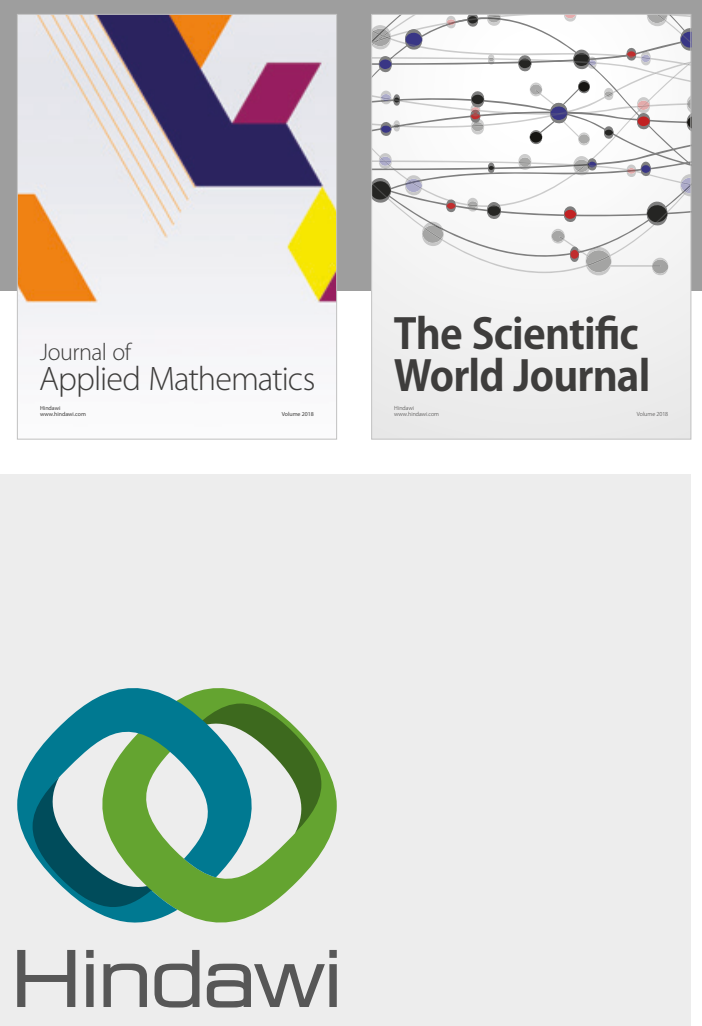

Submit your manuscripts at

www.hindawi.com

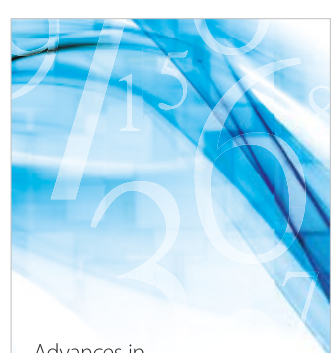

Advances in
Numerical Analysis
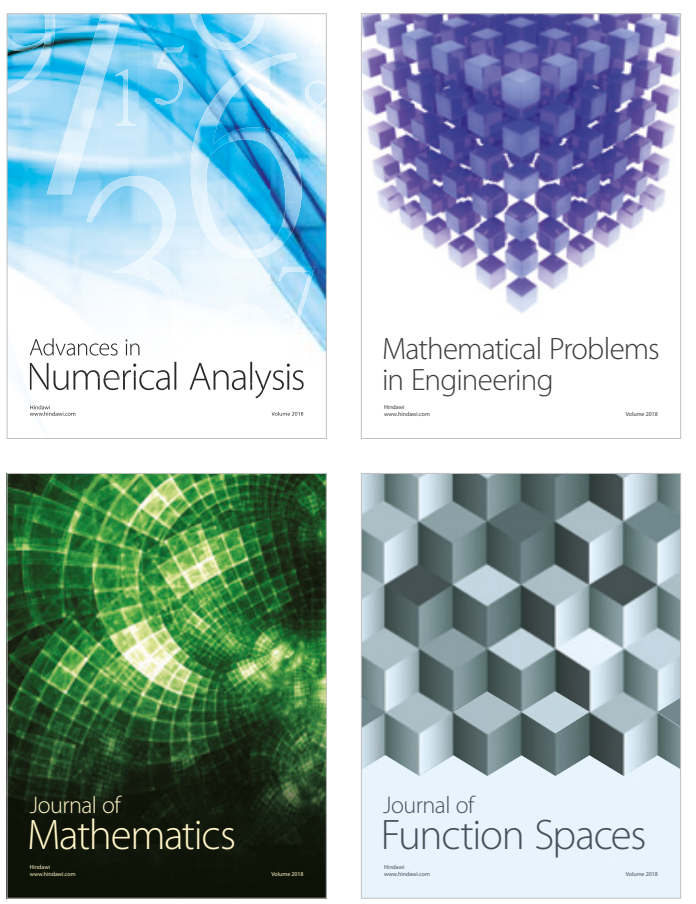

Mathematical Problems in Engineering

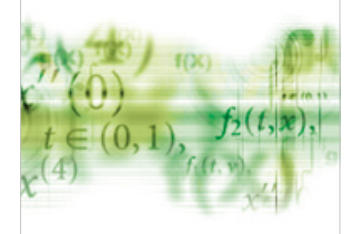

International Journal of

Differential Equations

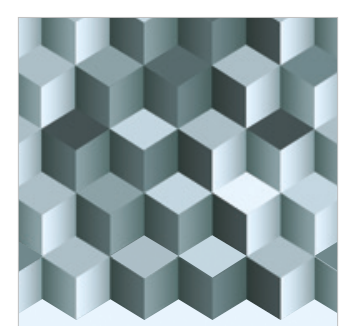

Journal of

Function Spaces
The Scientific

World Journal

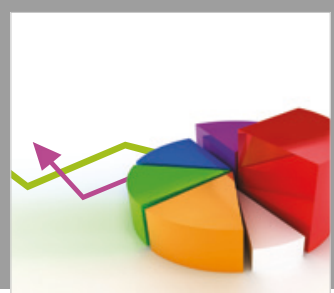

Journal of

Probability and Statistics
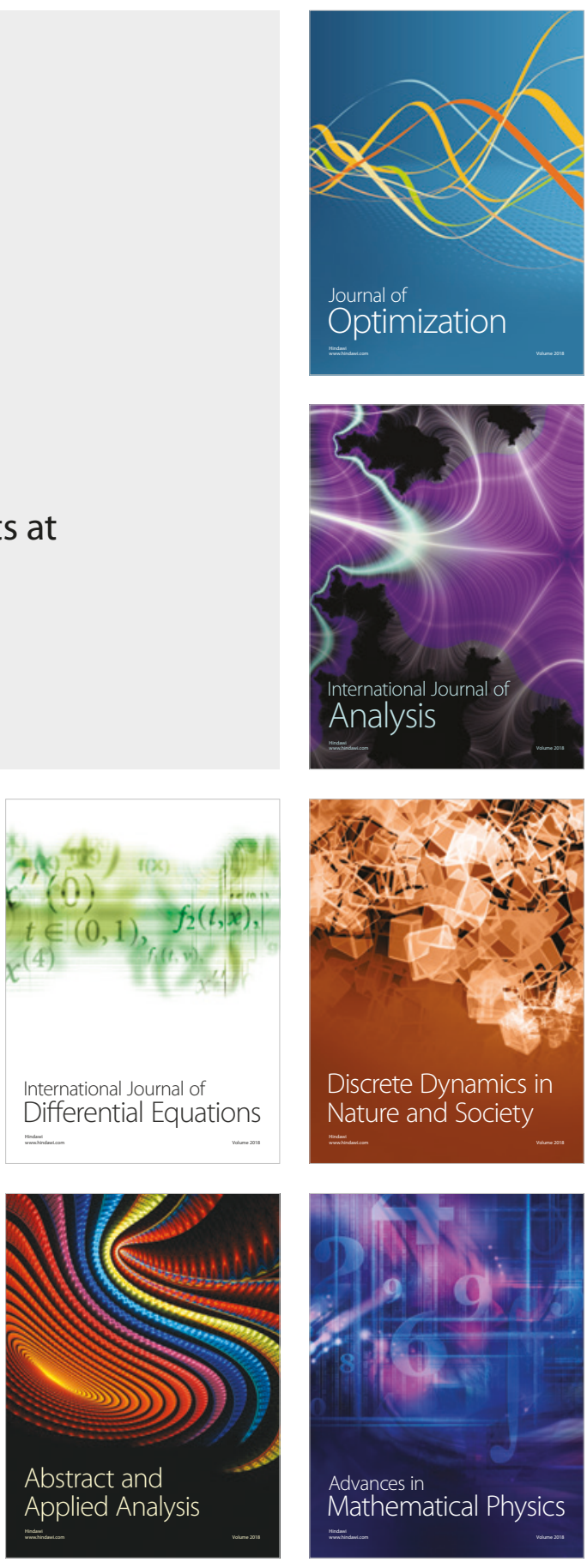